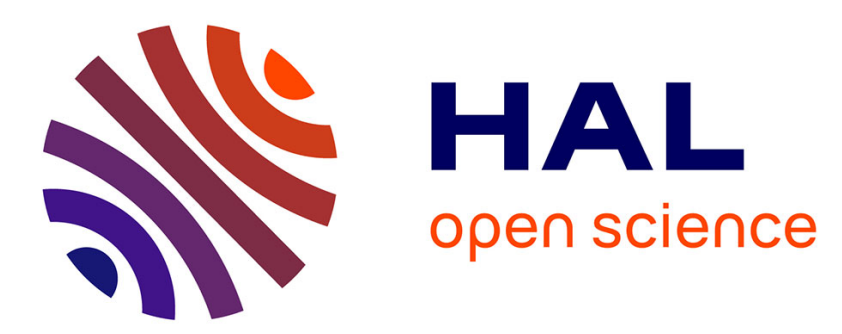

\title{
Detecting an obstacle immersed in a fluid by shape optimization methods
}

\author{
Mehdi Badra, Fabien Caubet, Marc Dambrine
}

\section{To cite this version:}

Mehdi Badra, Fabien Caubet, Marc Dambrine. Detecting an obstacle immersed in a fluid by shape optimization methods. Mathematical Models and Methods in Applied Sciences, 2011, 21 (10), pp.2069-

2101. hal-00583469

\author{
HAL Id: hal-00583469 \\ https://hal.science/hal-00583469
}

Submitted on 5 Apr 2011

HAL is a multi-disciplinary open access archive for the deposit and dissemination of scientific research documents, whether they are published or not. The documents may come from teaching and research institutions in France or abroad, or from public or private research centers.
L'archive ouverte pluridisciplinaire HAL, est destinée au dépôt et à la diffusion de documents scientifiques de niveau recherche, publiés ou non, émanant des établissements d'enseignement et de recherche français ou étrangers, des laboratoires publics ou privés. 


\title{
DETECTING AN OBSTACLE IMMERSED IN A FLUID BY SHAPE OPTIMIZATION METHODS
}

\author{
Mehdi BADRA \\ LMA, CNRS, Université de Pau et des Pays de l'Adour \\ av. de l'université, F-64013 Pau, France \\ mehdi.badra@univ-pau.fr \\ Fabien CAUBET \\ LMA, CNRS, Université de Pau et des Pays de l'Adour, \\ av. de l'université, F-64013 Pau, France \\ fabien.caubet@univ-pau.fr \\ Marc DAMBRINE \\ LMA, CNRS, Université de Pau et des Pays de l'Adour, \\ av. de l'université, F-64013 Pau, France \\ marc.dambrine@univ-pau.fr \\ Received (Day Month Year) \\ Revised (Day Month Year) \\ Communicated by (xxxxxxxxxx)
}

\begin{abstract}
The paper presents a theoretical study of an identification problem by shape optimization methods. The question is to detect an object immersed in a fluid. Here, the problem is modeled by the Stokes equations and treated as a nonlinear least-squares problem. We consider both the Dirichlet and Neumann boundary conditions. Firstly, we prove an identifiability result. Secondly, we prove the existence of the first order shape derivatives of the state, we characterize them and deduce the gradient of the least-squares functional. Moreover, we study the stability of this setting. We prove the existence of the second order shape derivatives and we give the expression of the shape Hessian. Finally, the compactness of the Riesz operator corresponding to this shape Hessian is shown and the ill-posedness of the identification problem follows. This explains the need of regularization to numerically solve this problem.
\end{abstract}

Keywords: stationary Stokes problem; shape sensitivity; geometric inverse problem.

AMS Subject Classification: 35R30,49Q10, 35Q30, (76D07).

\section{Introduction, notations and setting of the problem}

The detection and the reconstruction of an object immersed in a fluid is a source of many investigations. Recently, in 2005, Alvarez et al. studied in Ref. 4 the following inverse problem: an inaccessible rigid body $\omega$ is immersed in a viscous fluid, in such a way that $\omega$ plays the role of an obstacle around which the fluid is flowing in a 
greater bounded domain $\Omega$. They wish to determine $\omega$ (i.e. its shape and location) via boundary measurement on the boundary $\partial \Omega$. Under reasonable smoothness assumptions on $\Omega$ and $\omega$, they prove that one can identify $\omega$ via the measurement of both the velocity of the fluid and the Cauchy forces on some part of the boundary $\partial \Omega$. They also give a directional stability result for the inverse problem (see Theorem 1.3 in Ref. 4). After this first result, more investigations were conducted. In 2007, Heck et al. estimate in Ref. 19 the distance of a chosen point from the obstacle when the fluid motion is governed by the Stokes equations. In Ref. 5, Alves et al. use a method based on the analysis of a system of nonlinear integral equations to determine the shape and the location of a rigid body immersed in a viscous and incompressible fluid. In 2008, Conca et al. investigate in Ref. 11 the problem of the detection of a moving obstacle in a perfect fluid by a boundary measurement. They show that, when the obstacle is a ball, one may identify the position and the velocity of its center of mass from a single boundary measurement. In 2010, using complex analysis, Conca et al. prove in Ref. 12 that this result can not be generalized to any solid. However, they extend this one to moving ellipses and they proved that when the solid enjoys some symmetry properties, it can be "partially detected". Numerical experiments were also conducted: the numerical reconstruction is difficult as expected for an inverse problem.

This paper aims to explain these numerical difficulties. We focus on the question of stability of the shape optimization problem in the case of Stokes equations with Dirichlet and Neumann boundary conditions. Following previous works on electrical impedance tomography (by Eppler et al. in Ref. 14 and Afraites et al. in Ref. 2, Ref. 3), our strategy to solve this inverse problem is to minimize a least-squares functional. Then, we present the basis of the numerical analysis of this problem by characterizing the gradient of this functional. To study the stability, we compute the associated shape Hessian. We prove that this shape Hessian has a pathological behavior at possible solutions of the inverse problem: the Riesz operator corresponding to this shape Hessian is compact. This property explains why only simple shapes can be recovered. A result converging in the same direction was pointed out to us by one of the referee: this year, Ballerini proved in Ref. 8 a log-log stability estimate for a slightly different problem.

This paper is organized as follows. Firstly, we introduce the notations and the two problems we consider: the Dirichlet case, which is physically relevant, and the Neumann case much less physically relevant but more challenging from the mathematical point of view. Secondly, we state the main results of this work concerning these two cases in section 2. In particular, we provide an identification result, we prove the existence of the first order shape derivative of the state, we characterize this derivative and we give the expression of the gradient of the least-squares functional. Furthermore, we discuss higher order shape derivatives and we characterize the shape Hessian at a critical point. Finally, we justify the instability of the problem with the compactness of the Riesz operator corresponding to the shape 
Hessian at a critical shape. In section 3, we present some preliminary results: we recall an extension proved by Simon in Ref. 23 of the usual implicit functions theorem and we prove some results used in section 4 where we prove the main results of this work. The needed results on Stokes equations concerning the Neumann case (a theorem of existence and uniqueness of the solution and a local regularity result) are recalled in Appendix A.

Introduction of the general notations. Let us introduce the notations that we adopt in this paper. We denote by $\mathrm{L}^{p}, \mathrm{~W}^{m, p}$ and $\mathrm{H}^{s}$ the usual Lebesgue and Sobolev spaces. For $k \in \mathbb{N}$ and an open set $\Omega \subset \mathbb{R}^{N}(N=2$ or 3$)$, we denote by $C_{c}^{k}(\Omega)$ the space of functions with continuous $k$ first derivatives compactly supported in $\Omega$ and by $\|\cdot\|_{k, \infty}$ its natural norm. We also note by $\mathcal{D}(\Omega)$ the space of $C^{\infty}$ functions compactly supported in $\Omega$. We note in bold the vectorial functions and spaces: $\mathbf{L}^{p}$, $\mathbf{W}^{m, p}, \mathbf{H}^{s}, \ldots$ Moreover we will note respectively $\langle\cdot, \cdot\rangle_{\Omega}$ and $\langle\cdot, \cdot\rangle_{\partial \Omega}$ the dual product between $\left[\mathbf{H}^{1}(\Omega)\right]^{\prime}$ and $\mathbf{H}^{1}(\Omega)$ and the dual product between $\mathbf{H}^{-1 / 2}(\partial \Omega)$ and $\mathbf{H}^{1 / 2}(\partial \Omega)$. We denote by $|\Omega|$ the measure of $\Omega$ and by $\mathbb{1}_{O}$ the characteristic function of a measurable subset $O$. Moreover, $\mathbf{n}$ represents the external unit normal to $\partial \Omega$, and for a smooth enough function $u$, we note respectively $\partial_{\mathbf{n}} u$ and $\partial_{\mathbf{n n}}^{2} u$ the normal derivative and the second normal derivative of $u$. We also denote by $\mathcal{M}_{N, N}$ the space of the matrix of size $N \times N$. The tangential differential operators will be noted by the subscript $\Gamma$. In particular, for $\boldsymbol{w} \in \mathbf{W}^{1,1}(\partial \Omega)$ and $W \in W^{1,1}\left(\partial \Omega, \mathcal{M}_{N, N}\right)$, the following operators are defined on $\partial \Omega$ :

- $\nabla_{\Gamma} \boldsymbol{w}:=\nabla \boldsymbol{w}-(\nabla \boldsymbol{w} \mathbf{n}) \otimes \mathbf{n}$, where $\otimes$ denotes the tensorial product, (1.1)

- $\operatorname{div}_{\Gamma} W:=\operatorname{div} W-(\nabla W \mathbf{n}) \mathbf{n}$,

- $\Delta_{\Gamma} \boldsymbol{w}:=\operatorname{div}_{\Gamma}\left(\nabla_{\Gamma} \boldsymbol{w}\right)$, which is the Laplace-Beltrami operator.

For more details on tangential differential operators, we refer to Section 5.4.3 in Ref. 20.

The problem setting. Let $\Omega$ a bounded, connected and Lipschitz open subset of $\mathbb{R}^{N}$ (with $N=2$ or $N=3$ ). Let $\delta>0$ fixed (small). We define $\mathcal{O}_{\delta}$ the set of all open subsets $\omega$ of $\Omega$ with a $C^{2,1}$ boundary such that $\mathrm{d}(x, \partial \Omega)>\delta$ for all $x \in \omega$ and such that $\Omega \backslash \bar{\omega}$ is connected. We also define $\Omega_{\delta}$ an open set with a $C^{\infty}$ boundary and such that

$$
\{x \in \Omega ; \mathrm{d}(x, \partial \Omega)>\delta / 2\} \subset \Omega_{\delta} \subset\{x \in \Omega ; \mathrm{d}(x, \partial \Omega)>\delta / 3\} .
$$

Let $\boldsymbol{U}:=\left\{\boldsymbol{\theta} \in \mathbf{W}^{2, \infty}\left(\mathbb{R}^{N}\right)\right.$; Supp $\left.\boldsymbol{\theta} \subset \overline{\Omega_{\delta}}\right\}$ and $\mathcal{U}:=\left\{\boldsymbol{\theta} \in \boldsymbol{U} ;\|\boldsymbol{\theta}\|_{2, \infty}<1\right\}$ be the space of admissible deformations. If $\boldsymbol{\theta} \in \mathcal{U}$, then $(\boldsymbol{I}+\boldsymbol{\theta})$ is a diffeomorphism by Banach's fixed point Theorem. For such a $\boldsymbol{\theta}$ with Supp $\boldsymbol{\theta} \subset \Omega$ and $\omega \in \mathcal{O}_{\delta}$, we check $\Omega=(\mathbf{I}+\boldsymbol{\theta})(\Omega)$ and we define the perturbed domain $\omega_{\theta}:=(\mathbf{I}+\boldsymbol{\theta})(\omega)$ which is so that $\Omega \backslash \overline{\omega_{\theta}} \in \mathcal{O}_{\delta}$.

Let $\boldsymbol{f}_{\boldsymbol{b}}$ be an admissible boundary measurement and $O$ a non-empty open subset of $\partial \Omega$. We will study separately both the Dirichlet case and the Neumann case. 
The Dirichlet case. Let $\boldsymbol{g} \in \mathbf{H}^{5 / 2}(\partial \Omega)$ such that $\boldsymbol{g} \neq \mathbf{0}$ and satisfying the following condition:

$$
\int_{\partial \Omega} \boldsymbol{g} \cdot \mathbf{n}=0
$$

and let us consider, for $\omega \in \mathcal{O}_{\delta}$, the following overdetermined Stokes boundary values problem:

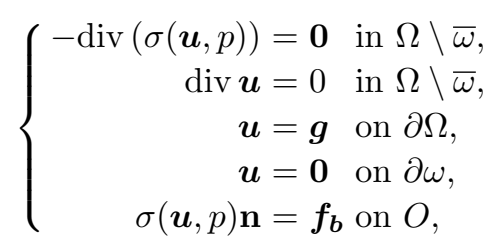

where $\sigma(\boldsymbol{u}, p):=\nu\left(\nabla \boldsymbol{u}+{ }^{t} \nabla \boldsymbol{u}\right)-p$ I is the stress tensor, with $\nu>0$ a given constant representing the kinematic viscosity of the liquid. Since $\operatorname{div} \boldsymbol{u}=0$ in $\Omega \backslash \bar{\omega}$, one has

$$
-\operatorname{div}(\sigma(\boldsymbol{u}, p))=-\nu \Delta \boldsymbol{u}+\nabla p,
$$

in $\Omega \backslash \bar{\omega}$ and we will use indifferently both expressions.

We suppose here that there exists $\omega$ such that (1.5) has a solution. This means that the measurement $f_{\boldsymbol{b}}$ is perfect, that is to say without error. Thus, we consider the following geometric inverse problem:

find $\omega \in \mathcal{O}_{\delta}$ and a pair $(\boldsymbol{u}, p)$ which satisfies the overdetermined system (1.5).

To solve this inverse problem, we consider, for $\omega \in \mathcal{O}_{\delta}$, the least-squares functional

$$
J_{D}(\omega):=\int_{O}\left|\sigma(\boldsymbol{u}(\omega), p(\omega)) \mathbf{n}-\boldsymbol{f}_{\boldsymbol{b}}\right|^{2},
$$

where $(\boldsymbol{u}(\omega), p(\omega)) \in \mathbf{H}^{1}(\Omega \backslash \bar{\omega}) \times \mathrm{L}^{2}(\Omega \backslash \bar{\omega})$ is a solution of the Stokes problem

$$
\left\{\begin{aligned}
-\operatorname{div}(\sigma(\boldsymbol{u}, p)) & =\mathbf{0} \text { in } \Omega \backslash \bar{\omega} \\
\operatorname{div} \boldsymbol{u} & =0 \text { in } \Omega \backslash \bar{\omega} \\
\boldsymbol{u} & =\boldsymbol{g} \text { on } \partial \Omega, \\
\boldsymbol{u} & =\mathbf{0} \text { on } \partial \omega .
\end{aligned}\right.
$$

Since we imposed the compatibility condition (1.4), problem (1.7) has a unique solution once a normalization condition on the pressure $p$ is imposed (see for example Chapter 1 in Ref. 24). This solution will be called the state. Here, we choose the normalization

$$
\int_{O}(\sigma(\boldsymbol{u}, p) \mathbf{n}) \cdot \mathbf{n}=\int_{O} \boldsymbol{f}_{\boldsymbol{b}} \cdot \mathbf{n}
$$

Then, we try to minimize the least-squares criterion $J_{D}$ :

$$
\omega^{*}=\underset{\omega \in \mathcal{O}_{\delta}}{\operatorname{argmin}} J_{D}(\omega) .
$$

Indeed, if $\omega^{*}$ is solution of the inverse problem (1.6), then $J\left(\omega^{*}\right)=0$ and (1.8) holds. Conversely, if $\omega^{*}$ solves (1.8) with $J\left(\omega^{*}\right)=0$, then this domain $\omega^{*}$ is a solution of the inverse problem. 
The Neumann case. We mimic the study of the Dirichlet case. Let $\boldsymbol{f} \in \mathbf{H}^{3}\left(\mathbb{R}^{N}\right)$ such that $\boldsymbol{f} \neq \mathbf{0}, \boldsymbol{f} \equiv \mathbf{0}$ in $\Omega_{\delta}$ and satisfying the condition:

$$
\int_{\Omega} f=0 .
$$

For $\omega \in \mathcal{O}_{\delta}$, we consider the overdetermined Stokes boundary values problem:

$$
\left\{\begin{aligned}
-\nu \Delta \boldsymbol{u}+\nabla p=\boldsymbol{f} & \text { in } \Omega \backslash \bar{\omega} \\
\operatorname{div} \boldsymbol{u}=0 & \text { in } \Omega \backslash \bar{\omega} \\
-\nu \partial_{\mathbf{n}} \boldsymbol{u}+p \mathbf{n}=\mathbf{0} & \text { on } \partial \Omega \\
-\nu \partial_{\mathbf{n}} \boldsymbol{u}+p \mathbf{n}=\mathbf{0} & \text { on } \partial \omega \\
\boldsymbol{u} & =\boldsymbol{f}_{\boldsymbol{b}} \text { on } O
\end{aligned}\right.
$$

where $\nu>0$ is a given constant representing the kinematic viscosity of the liquid.

We suppose here that there exists $\omega$ such that (1.10) has a solution. This means that the measurement $f_{b}$ is perfect. Thus, we consider the geometric inverse problem:

find $\omega \in \mathcal{O}_{\delta}$ and a pair $(\boldsymbol{u}, p)$ which satisfies the overdetermined system (1.10).

To study this inverse problem, we introduce, for $\omega \in \mathcal{O}_{\delta}$, the least-squares functional

$$
J(\omega):=\int_{O}\left|\boldsymbol{u}(\omega)-\boldsymbol{f}_{\boldsymbol{b}}\right|^{2},
$$

where $(\boldsymbol{u}(\omega), p(\omega)) \in \mathbf{H}^{1}(\Omega \backslash \bar{\omega}) \times \mathrm{L}^{2}(\Omega \backslash \bar{\omega})$ satisfies the Stokes boundary values problem

$$
\left\{\begin{aligned}
-\nu \Delta \boldsymbol{u}+\nabla p & =\boldsymbol{f} \text { in } \Omega \backslash \bar{\omega} \\
\operatorname{div} \boldsymbol{u} & =0 \text { in } \Omega \backslash \bar{\omega} \\
-\nu \partial_{\mathbf{n}} \boldsymbol{u}+p \mathbf{n} & =\mathbf{0} \text { on } \partial \Omega \\
-\nu \partial_{\mathbf{n}} \boldsymbol{u}+p \mathbf{n} & =\mathbf{0} \text { on } \partial \omega .
\end{aligned}\right.
$$

Since $\boldsymbol{f}$ satisfies the condition (1.9) and since $\boldsymbol{f} \equiv \mathbf{0}$ in $\Omega_{\delta}$, the compatibility condition of problem (1.12) is automatically satisfied. According to Appendix A.1, it has a unique solution once a normalization condition on $\boldsymbol{u}$ is imposed. Here, we take

$$
\int_{O} u=\int_{O} f_{b}
$$

Then, we try to minimize the least-squares criterion $J$ :

$$
\omega^{*}=\underset{\omega \in \mathcal{O}_{\delta}}{\operatorname{argmin}} J(\omega) .
$$

Indeed, if $\omega^{*}$ is solution of the inverse problem (1.11), then $J\left(\omega^{*}\right)=0$ and (1.13) holds. Conversely, if $\omega^{*}$ solves (1.13) with $J\left(\omega^{*}\right)=0$, then this domain $\omega^{*}$ is a solution of the inverse problem.

Remark 1.1. In this Neumann case, we can also consider a non-homogeneous Neumann data of the kind $-\nu \partial_{\mathbf{n}} \boldsymbol{u}+p \mathbf{n}=\boldsymbol{g}$ on $\partial \Omega$ instead of / or in addition to 
the force $\boldsymbol{f}$. In the Dirichlet case, we can also consider a force $\boldsymbol{f}$ with homogeneous Dirichlet data on $\partial \Omega$. These modifications do not change the conclusions of the work but complicate the notations and computations.

Introduction of the needed functional tools. Let $T>0$, that we will have to fix small. We will use the shape calculus introduced by Murat and Simon in Ref. 22 . Thus, we consider the function

$$
\phi: t \in[0, T) \mapsto \mathbf{I}+t \boldsymbol{V} \in \mathbf{W}^{2, \infty}\left(\mathbb{R}^{N}\right) .
$$

where $\boldsymbol{V} \in \boldsymbol{U}$. Note that for small $t, \phi(t)$ is a diffeomorphism of $\mathbb{R}^{N}$ and that $\phi^{\prime}(0)=\boldsymbol{V}$ vanishes on $\partial \Omega$ and even on the tubular neighborhood $\Omega \backslash \overline{\Omega_{\delta}}$ of $\partial \Omega$. For $t \in[0, T)$, we define $\omega_{t}:=\phi(t)(\omega)$ where $\phi$ is defined by (1.14). For the rest of the paper, we use a subscript " $t$ " to indicate that the quantity is defined on the time $t$ dependent domain. For instance, $\mathbf{n}_{t}$ is the external unit normal of $\Omega \backslash \overline{\omega_{t}}$.

\section{Statement of the main results}

We will state the main results in the two cases we study: the Dirichlet and the Neumann cases exposed in the previous section. We obtain similar results.

\subsection{The Dirichlet case}

Identifiability result We first quote an identifiability result in the Dirichlet case (see Theorem 1.2 in Ref. 4) proved by Alvarez et al:: it states that given a fixed $\boldsymbol{g}$, two different geometries $\omega_{0}$ and $\omega_{1}$ in $\mathcal{O}_{\delta}$ yield two different measures $\boldsymbol{f}_{\boldsymbol{b} \mathbf{1}}$ and $\boldsymbol{f}_{\boldsymbol{b} \mathbf{2}}$. Hence problem (1.6) admits a unique solution.

Theorem 2.1 (C. Alvarez, C. Conca, L. Friz, O. Kavian, J.H. Ortega, Ref. 4). Let $\Omega \subseteq \mathbb{R}^{N}, N=2$ or $N=3$, be a bounded Lipschitz domain, and $O$ a non-empty open subset of $\partial \Omega$. Let

$$
\omega_{0}, \omega_{1} \in \mathcal{D}_{\mathrm{ad}}:=\{\omega \subset \subset \Omega ; \omega \text { is open, Lipschitz and } \Omega \backslash \bar{\omega} \text { is connected }\}
$$

and $\boldsymbol{g} \in \mathbf{H}^{3 / 2}(\partial \Omega)$ with $\boldsymbol{g} \neq \mathbf{0}$, satisfying the flux condition (1.4). For $\epsilon_{*}=0$ or $\epsilon_{*}=1$, let $\left(\boldsymbol{u}_{\boldsymbol{j}}, p_{j}\right)$ for $j=0,1$, be a solution of

$$
\left\{\begin{aligned}
-\operatorname{div}\left(\sigma\left(\boldsymbol{u}_{\boldsymbol{j}}, p_{j}\right)\right)+\epsilon_{*} \operatorname{div}\left(\boldsymbol{u}_{\boldsymbol{j}} \otimes \boldsymbol{u}_{\boldsymbol{j}}\right) & =\mathbf{0} \text { in } \Omega \backslash \overline{\omega_{j}}, \\
\operatorname{div} \boldsymbol{u}_{\boldsymbol{j}} & =0 \text { in } \Omega \backslash \overline{\omega_{j}}, \\
\boldsymbol{u}_{\boldsymbol{j}} & =\boldsymbol{g} \text { on } \partial \Omega \\
\boldsymbol{u}_{\boldsymbol{j}} & =\mathbf{0} \text { on } \partial \omega_{j} .
\end{aligned}\right.
$$

Assume that $\left(\boldsymbol{u}_{\boldsymbol{j}}, p_{j}\right)$ are such that

$$
\sigma\left(\boldsymbol{u}_{j}, p_{j}\right) \mathbf{n}=\sigma\left(\boldsymbol{u}_{\boldsymbol{j}}, p_{j}\right) \mathbf{n} \quad \text { on } O
$$

Then $\omega_{0} \equiv \omega_{1}$. 
Sensitivity with respect to the domain Secondly, we aim to make a sensitivity (with respect to the shape) analysis. The Stokes problem on $\Omega \backslash \overline{\omega_{t}}$

$$
\left\{\begin{aligned}
-\operatorname{div}\left(\sigma\left(\boldsymbol{u}_{\boldsymbol{t}}, p_{t}\right)\right) & =\mathbf{0} \text { in } \Omega \backslash \overline{\omega_{t}} \\
\operatorname{div} \boldsymbol{u}_{\boldsymbol{t}} & =0 \text { in } \Omega \backslash \overline{\omega_{t}} \\
\boldsymbol{u}_{\boldsymbol{t}} & =\boldsymbol{g} \text { on } \partial \Omega \\
\boldsymbol{u}_{\boldsymbol{t}} & =\mathbf{0} \text { on } \partial \omega_{t}
\end{aligned}\right.
$$

admits a unique solution $\left(\boldsymbol{u}_{\boldsymbol{t}}, p_{t}\right) \in \mathbf{H}^{1}\left(\Omega \backslash \overline{\omega_{t}}\right) \times \mathrm{L}^{2}\left(\Omega \backslash \overline{\omega_{t}}\right)$ satisfying the normalization condition

$$
\int_{O}\left(\sigma\left(\boldsymbol{u}_{\boldsymbol{t}}, p_{t}\right) \mathbf{n}\right) \cdot \mathbf{n}=\int_{O} \boldsymbol{f}_{\boldsymbol{b}} \cdot \mathbf{n} .
$$

Proposition 2.1 (First order shape derivatives of the state). The solution $(\boldsymbol{u}, p)$ is differentiable with respect to the domain. Moreover, the derivatives $\boldsymbol{u}^{\prime}$ and $p^{\prime}$ belong to $\mathbf{H}^{2}\left(\Omega_{\delta} \backslash \bar{\omega}\right)$ and to $\mathrm{H}^{1}\left(\Omega_{\delta} \backslash \bar{\omega}\right)$. The pair $\left(\boldsymbol{u}^{\prime}, p^{\prime}\right) \in \mathbf{H}^{1}(\Omega \backslash \bar{\omega}) \times \mathrm{L}^{2}(\Omega \backslash \bar{\omega})$ is the only solution of the following boundary values problem

$$
\left\{\begin{aligned}
-\operatorname{div}\left(\sigma\left(\boldsymbol{u}^{\prime}, p^{\prime}\right)\right) & =\mathbf{0} & & \text { in } \Omega \backslash \bar{\omega} \\
\operatorname{div} \boldsymbol{u}^{\prime} & =0 & & \text { in } \Omega \backslash \bar{\omega} \\
\boldsymbol{u}^{\prime} & =\mathbf{0} & & \text { on } \partial \Omega, \\
\boldsymbol{u}^{\prime} & =-\nabla \boldsymbol{u} \boldsymbol{V} & & \text { on } \partial \omega,
\end{aligned}\right.
$$

with the normalization condition

$$
\int_{O}\left(\sigma\left(\boldsymbol{u}^{\prime}, p^{\prime}\right) \mathbf{n}\right) \cdot \mathbf{n}=0
$$

Proposition 2.2 (First order shape derivatives of the functional). For $\boldsymbol{V}$ in $\boldsymbol{U}$, the least-squares functional $J_{D}$ is differentiable at $\boldsymbol{\omega}$ in the direction $\boldsymbol{V}$ with

$$
\mathrm{D} J_{D}(\omega) \cdot \boldsymbol{V}=-\int_{\partial \omega}\left[(\sigma(\boldsymbol{w}, q) \mathbf{n}) \cdot \partial_{\mathbf{n}} \boldsymbol{u}\right](\boldsymbol{V} \cdot \mathbf{n}),
$$

where $(\boldsymbol{w}, q) \in \mathbf{H}^{1}(\Omega \backslash \bar{\omega}) \times \mathrm{L}^{2}(\Omega \backslash \bar{\omega})$ is the solution of the following Stokes boundary values problem:

$$
\left\{\begin{aligned}
-\operatorname{div}(\sigma(\boldsymbol{w}, q)) & =\mathbf{0} & & \text { in } \Omega \backslash \bar{\omega}, \\
\operatorname{div} \boldsymbol{w} & =0 & & \text { in } \Omega \backslash \bar{\omega}, \\
\boldsymbol{w} & =2\left(\sigma(\boldsymbol{u}, p) \mathbf{n}-\boldsymbol{f}_{\boldsymbol{b}}\right) \mathbb{1}_{O} & & \text { on } \partial \Omega, \\
\boldsymbol{w} & =\mathbf{0} & & \text { on } \partial \omega,
\end{aligned}\right.
$$

with the normalization condition

$$
\int_{O}(\sigma(\boldsymbol{w}, q) \mathbf{n}) \cdot \mathbf{n}=0
$$

Remark 2.1. The differentiability with respect to the domain of $\boldsymbol{u}$ and $p$ as well as Proposition 2.2 remain true under weaker assumptions. Indeed, the proof is still valid with $\boldsymbol{g} \in \mathbf{H}^{1 / 2}(\partial \Omega)$ and $\omega$ with a $C^{1,1}$ boundary (but in this case, the expression of $\mathrm{D} J_{D}(\omega) \cdot \boldsymbol{V}$ has to be seen as a duality product $\mathrm{H}^{-1 / 2} \times \mathrm{H}^{1 / 2}$ ). 
Second order analysis: justification of the instability Finally, we want to study the stability of the optimization problem (1.8) at $\omega^{*}$.

Proposition 2.3 (Characterization of the shape Hessian at a critical shape). The solution $(\boldsymbol{u}, p)$ is twice differentiable with respect to the domain. Moreover, for $\boldsymbol{V} \in \boldsymbol{U}$, we have

$$
\mathrm{D}^{2} J_{D}\left(\omega^{*}\right) \cdot \boldsymbol{V} \cdot \boldsymbol{V}=\int_{\partial \omega^{*}}-\left(\sigma\left(\boldsymbol{w}^{\prime}, q^{\prime}\right) \mathbf{n}\right) \cdot(\nabla \boldsymbol{u} \boldsymbol{V}),
$$

where $\left(\boldsymbol{w}^{\prime}, q^{\prime}\right) \in \mathbf{H}^{1}\left(\Omega \backslash \overline{\omega^{*}}\right) \times \mathrm{L}^{2}\left(\Omega \backslash \overline{\omega^{*}}\right)$ is the solution of the following problem:

$$
\left\{\begin{array}{rlrl}
-\operatorname{div}\left(\sigma\left(\boldsymbol{w}^{\prime}, q^{\prime}\right)\right) & =\mathbf{0} & & \text { in } \Omega \backslash \overline{\omega^{*},} \\
\operatorname{div} \boldsymbol{w}^{\prime} & =0 & & \text { in } \Omega \backslash \overline{\omega^{*}}, \\
\boldsymbol{w}^{\prime} & =2 \sigma\left(\boldsymbol{u}^{\prime}, p^{\prime}\right) \mathbf{n} \mathbb{1}_{O} & \text { on } \partial \Omega, \\
\boldsymbol{w}^{\prime} & =\mathbf{0} & & \text { on } \partial \omega^{*},
\end{array}\right.
$$

with the normalization condition

$$
\int_{O}\left(\sigma\left(\boldsymbol{w}^{\prime}, q^{\prime}\right) \mathbf{n}\right) \cdot \mathbf{n}=0 .
$$

Proposition 2.4 (Compactness at a critical point). The Riesz operator corresponding to $\mathrm{D}^{2} J_{D}\left(\omega^{*}\right)$ defined from $\mathbf{H}^{1 / 2}\left(\partial \omega^{*}\right)$ to $\mathbf{H}^{-1 / 2}\left(\partial \omega^{*}\right)$ is compact.

Remark 2.2. We refer to Theorem IV-5-1 in Ref. 17 for the local regularity result for the solutions in the Dirichlet case. This point is crucial for proving Proposition 2.4 with the method we adopt here.

\subsection{The Neumann case}

Identifiability result We first present a new identifiability result for the Neumann case.

Theorem 2.2 (Identifiability result). Let $\Omega \subseteq \mathbb{R}^{N}, N=2$ or $N=3$, be a bounded Lipschitz domain and $O$ a non-empty open subset of $\partial \Omega$. Let $\omega_{0}, \omega_{1} \in \mathcal{O}_{\delta}$ and $\boldsymbol{f} \in \mathbf{L}^{2}(\Omega)$ with $\boldsymbol{f} \neq \mathbf{0}$ and $\boldsymbol{f} \equiv \mathbf{0}$ in $\Omega_{\delta}$ satisfying the condition (1.9). Let $\left(\boldsymbol{u}_{\boldsymbol{j}}, p_{j}\right)$, for $j=0,1$, be a solution of

$$
\left\{\begin{aligned}
-\nu \Delta \boldsymbol{u}_{j}+\nabla p_{j} & =\boldsymbol{f} \text { in } \Omega \backslash \overline{\omega_{j}} \\
\operatorname{div} \boldsymbol{u}_{j} & =0 \text { in } \Omega \backslash \overline{\omega_{j}} \\
-\nu \partial_{\mathbf{n}} \boldsymbol{u}_{j}+p_{j} \mathbf{n} & =\mathbf{0} \text { on } \partial \Omega \\
-\nu \partial_{\mathbf{n}_{\mathbf{j}}} \boldsymbol{u}_{\boldsymbol{j}}+p_{j} \mathbf{n}_{\boldsymbol{j}} & =\mathbf{0} \text { on } \partial \omega_{j}
\end{aligned}\right.
$$

Assume that $\left(\boldsymbol{u}_{\boldsymbol{j}}, p_{j}\right)$ are such that $\boldsymbol{u}_{\mathbf{0}}=\boldsymbol{u}_{\mathbf{1}}$ on $O$. Then $\omega_{0} \equiv \omega_{1}$.

Remark 2.3. This proof of identifiability will be done under the weaker assumption: $\omega_{0}$ and $\omega_{1}$ have a Lipschitz boundary. 
Sensitivity with respect to the domain. Secondly, we aim to make a sensitivity (with respect to the shape) analysis, as in the Dirichlet case. The Stokes problem on $\Omega \backslash \overline{\omega_{t}}$

$$
\left\{\begin{aligned}
-\nu \Delta \boldsymbol{u}_{t}+\nabla p_{t} & =\boldsymbol{f} \text { in } \Omega \backslash \overline{\omega_{t}} \\
\operatorname{div} \boldsymbol{u}_{\boldsymbol{t}}=0 & \text { in } \Omega \backslash \overline{\omega_{t}} \\
-\nu \partial_{\mathbf{n}} \boldsymbol{u}_{\boldsymbol{t}}+p_{t} \mathbf{n}=\mathbf{0} & \text { on } \partial \Omega \\
-\nu \partial_{\mathbf{n}_{t}} \boldsymbol{u}_{\boldsymbol{t}}+p_{t} \mathbf{n}_{\boldsymbol{t}}=\mathbf{0} & \text { on } \partial \omega_{t}
\end{aligned}\right.
$$

admits a unique solution $\left(\boldsymbol{u}_{\boldsymbol{t}}, p_{t}\right) \in \mathbf{H}^{1}\left(\Omega \backslash \overline{\omega_{t}}\right) \times \mathrm{L}^{2}\left(\Omega \backslash \overline{\omega_{t}}\right)$ satisfying the normalization condition

$$
\int_{O} \boldsymbol{u}_{\boldsymbol{t}}=\int_{O} \boldsymbol{f}_{\boldsymbol{b}}
$$

Notice that the compatibility condition of problem (2.1) is automatically satisfied since $\boldsymbol{f}$ satisfies the condition (1.9) and since $\boldsymbol{f} \equiv \mathbf{0}$ in $\Omega_{\delta}$.

Proposition 2.5 (First order shape derivatives of the state). The solution $(\boldsymbol{u}, p)$ is differentiable with respect to the domain. Moreover, the shape derivatives $\boldsymbol{u}^{\prime}$ and $p^{\prime}$ belong to $\mathbf{H}^{2}\left(\Omega_{\delta} \backslash \bar{\omega}\right)$ and to $\mathrm{H}^{1}\left(\Omega_{\delta} \backslash \bar{\omega}\right)$. The pair $\left(\boldsymbol{u}^{\prime}, p^{\prime}\right) \in \mathbf{H}^{1}(\Omega \backslash \bar{\omega}) \times \mathrm{L}^{2}(\Omega \backslash \bar{\omega})$ is the only solution of the following boundary values problem

$$
\left\{\begin{aligned}
-\nu \Delta \boldsymbol{u}^{\prime}+\nabla p^{\prime} & =\mathbf{0} \text { in } \Omega \backslash \bar{\omega} \\
\operatorname{div} \boldsymbol{u}^{\prime} & =0 \text { in } \Omega \backslash \bar{\omega} \\
-\nu \partial_{\mathbf{n}} \boldsymbol{u}^{\prime}+p^{\prime} \mathbf{n} & =\mathbf{0} \text { on } \partial \Omega \\
-\nu \partial_{\mathbf{n}} \boldsymbol{u}^{\prime}+p^{\prime} \mathbf{n} & =\left(\nu \partial_{\mathbf{n n}}^{2} \boldsymbol{u}-\partial_{\mathbf{n}} p \mathbf{n}\right)(\boldsymbol{V} \cdot \mathbf{n})+p \nabla_{\Gamma}(\boldsymbol{V} \cdot \mathbf{n})-\nu \nabla \boldsymbol{u} \nabla_{\Gamma}(\boldsymbol{V} \cdot \mathbf{n}) \text { on } \partial \omega
\end{aligned}\right.
$$

with the normalization condition

$$
\int_{O} \boldsymbol{u}^{\prime}=\mathbf{0}
$$

Remark 2.4 (Meaning of high order derivatives on $\partial \omega$ ). By the local regularity result Appendix A.2, $\boldsymbol{u} \in \mathbf{H}^{3}\left(\Omega_{\delta} \backslash \bar{\omega}\right), p \in \mathrm{H}^{2}\left(\Omega_{\delta} \backslash \bar{\omega}\right)$, then the quantity $\nu \partial_{\mathbf{n n}}^{2} \boldsymbol{u}-\partial_{\mathbf{n}} p \mathbf{n}$ belongs to $\mathbf{H}^{1 / 2}(\partial \omega)$.

Proposition 2.6 (First order shape derivatives of the functional). For $\boldsymbol{V}$ in $\boldsymbol{U}$, the least-squares functional $J$ is differentiable at $\omega$ in the direction $\boldsymbol{V}$ with

$$
\mathrm{D} J(\omega) \cdot \boldsymbol{V}=\int_{\partial \omega}\left[\left(\nu \partial_{\mathbf{n n}}^{2} \boldsymbol{u}-\partial_{\mathbf{n}} p \mathbf{n}\right) \cdot \boldsymbol{w}-\operatorname{div}_{\Gamma}\left(\left(p \mathrm{I}-\nu^{t} \nabla \boldsymbol{u}\right) \boldsymbol{w}\right)\right](\boldsymbol{V} \cdot \mathbf{n}),
$$

where $(\boldsymbol{w}, q) \in \mathbf{H}^{1}(\Omega \backslash \bar{\omega}) \times \mathrm{L}^{2}(\Omega \backslash \bar{\omega})$ is the solution of the following problem:

$$
\left\{\begin{aligned}
-\nu \Delta \boldsymbol{w}+\nabla q & =\mathbf{0} & & \text { in } \Omega \backslash \bar{\omega} \\
\operatorname{div} \boldsymbol{w} & =0 & & \text { in } \Omega \backslash \bar{\omega} \\
-\nu \partial_{\mathbf{n}} \boldsymbol{w}+q \mathbf{n} & =2\left(\boldsymbol{u}-\boldsymbol{f}_{\boldsymbol{b}}\right) \mathbb{1}_{O} & & \text { on } \partial \Omega \\
-\nu \partial_{\mathbf{n}} \boldsymbol{w}+q \mathbf{n} & =\mathbf{0} & & \text { on } \partial \omega
\end{aligned}\right.
$$


with the normalization condition

$$
\int_{O} \boldsymbol{w}=\mathbf{0}
$$

Remark 2.5 (Meaning of high order derivatives on $\partial \omega$ ). The expression of shape derivative involves high order derivatives of the couple $(\boldsymbol{u}, p)$ that have to be well defined. By the local regularity result Appendix A.2, $\boldsymbol{w} \in \mathbf{H}^{3}\left(\Omega_{\delta} \backslash \bar{\omega}\right)$. It follows that $\boldsymbol{w} \in \mathbf{H}^{5 / 2}(\partial \omega)$ and $p \mathrm{I}-\nu^{t} \nabla \boldsymbol{u} \in \mathrm{H}^{3 / 2}(\partial \omega)$. Therefore, the function $\left(p \mathrm{I}-\nu^{t} \nabla \boldsymbol{u}\right) \boldsymbol{w} \in \mathbf{W}^{1,1}(\partial \omega)$ has a tangential divergence on $\partial \omega$.

Moreover, using the Sobolev embeddings in dimension $N=2$ or $N=3$,

$$
\boldsymbol{w} \in \mathbf{H}^{3}\left(\Omega_{\delta} \backslash \bar{\omega}\right) \hookrightarrow \mathbf{L}^{\infty}\left(\Omega_{\delta} \backslash \bar{\omega}\right) \quad \text { and } \quad \nabla \boldsymbol{w} \in \mathrm{H}^{2}\left(\Omega_{\delta} \backslash \bar{\omega}\right) \hookrightarrow \mathrm{L}^{\infty}\left(\Omega_{\delta} \backslash \bar{\omega}\right) .
$$

Thus, $\boldsymbol{w} \in \mathbf{W}^{2, \infty}\left(\Omega_{\delta} \backslash \bar{\omega}\right)$, then $\left(p \mathrm{I}-\nu^{t} \nabla \boldsymbol{u}\right) \boldsymbol{w} \in \mathbf{H}^{1}\left(\Omega_{\delta} \backslash \bar{\omega}\right)$ and finally we get that $\operatorname{div}_{\Gamma}\left(p \mathrm{I}-\nu^{t} \nabla \boldsymbol{u}\right) \boldsymbol{w} \in \mathrm{H}^{-1 / 2}(\partial \omega)$.

Remark 2.6. The differentiability with respect to the domain of $\boldsymbol{u}$ and $p$ as well as Proposition 2.6 remain true under weaker assumptions. Indeed, the proof is still valid with $\boldsymbol{f} \in \mathbf{H}^{1}\left(\mathbb{R}^{N}\right)$ and $\omega$ with a $C^{1,1}$ boundary (but in this case, the expression of $\mathrm{D} J(\omega) \cdot \boldsymbol{V}$ has to be seen as a duality product $\left.\mathrm{H}^{-1 / 2} \times \mathrm{H}^{1 / 2}\right)$.

Second order analysis: justification of the instability. Finally, we study the stability of the optimization problem (1.13) at $\omega^{*}$.

Proposition 2.7 (Characterization of the shape Hessian at a critical shape). The solution $(\boldsymbol{u}, p)$ is twice differentiable with respect to the domain. Moreover, for $\boldsymbol{V} \in \boldsymbol{U}$, we have

$\mathrm{D}^{2} J\left(\omega^{*}\right) \cdot \boldsymbol{V} \cdot \boldsymbol{V}=\int_{\partial \omega^{*}}\left[\left(\nu \partial_{\mathbf{n n}}^{2} \boldsymbol{u}-\partial_{\mathbf{n}} p \mathbf{n}\right)(\boldsymbol{V} \cdot \mathbf{n})+p \nabla_{\Gamma}(\boldsymbol{V} \cdot \mathbf{n})-\nu \nabla \boldsymbol{u} \nabla_{\Gamma}(\boldsymbol{V} \cdot \mathbf{n})\right] \cdot \boldsymbol{w}^{\prime}$, where $\left(\boldsymbol{w}^{\prime}, q^{\prime}\right) \in \mathbf{H}^{1}\left(\Omega \backslash \overline{\omega^{*}}\right) \times \mathrm{L}^{2}\left(\Omega \backslash \overline{\omega^{*}}\right)$ satisfies the following Stokes boundary values problem:

$$
\left\{\begin{aligned}
-\nu \Delta \boldsymbol{w}^{\prime}+\nabla q^{\prime} & =\mathbf{0} & & \text { in } \Omega \backslash \overline{\omega^{*},} \\
\operatorname{div} \boldsymbol{w}^{\prime} & =0 & & \text { in } \Omega \backslash \overline{\omega^{*},} \\
-\nu \partial_{\mathbf{n}} \boldsymbol{w}^{\prime}+q^{\prime} \mathbf{n} & =2 \boldsymbol{u}^{\prime} \mathbb{1}_{O} & & \text { on } \partial \Omega, \\
-\nu \partial_{\mathbf{n}} \boldsymbol{w}^{\prime}+q^{\prime} \mathbf{n} & =\mathbf{0} & & \text { on } \partial \omega^{*},
\end{aligned}\right.
$$

with the normalization condition

$$
\int_{O} \boldsymbol{w}^{\prime}=\mathbf{0}
$$

Proposition 2.8 (Compactness at a critical point). The Riesz operator corresponding to $\mathrm{D}^{2} J\left(\omega^{*}\right)$ defined from $\mathbf{H}^{1 / 2}\left(\partial \omega^{*}\right)$ to $\mathbf{H}^{-1 / 2}\left(\partial \omega^{*}\right)$ is compact. 


\subsection{About the lack of stability.}

Statements 2.4 and 2.8 explain the difficulties encountered to solve numerically this problem. Indeed, the gradient has not a uniform sensitivity with respect to the deformation directions. Since $J$ is twice differentiable, it behaves about $\omega^{*}$ as its second order approximation. the compactness result means, roughly speaking, that in a neighborhood of $\omega^{*}$ (i.e. for $t$ small) one cannot expect an estimate of the kind $C t \leq \sqrt{J\left(\omega_{t}\right)}$ with a constant $C$ uniform in $\boldsymbol{V}$.

However we prove that, for a finite dimensional space of deformation fields, the discrete shape Hessian is coercive: our identifiability result proves that the domain to be recovered is a local strict minimum of the least-squares functional. Let us take the example of a starshaped domain $\omega$ in dimension two. Assume that $\partial \omega$ is parametrized by

$$
\begin{gathered}
\partial \omega=\left\{\left(\begin{array}{l}
g_{0} \\
g_{1}
\end{array}\right)+\left(g_{2}+\sum_{k=1}^{\infty}\left(g_{2 k+1} \cos (k t)+g_{2 k+2} \sin (k t)\right)\right)\left(\begin{array}{c}
\cos (t) \\
\sin (t)
\end{array}\right)\right. \\
\left.=\sum_{k=0}^{\infty} g_{k} \boldsymbol{V}_{k}(t) ; t \in(0,2 \pi)\right\},
\end{gathered}
$$

where $g_{k} \in \mathbb{R}$. Then, for all $n \in \mathbb{N}$, we have an estimate of the kind

$$
\forall \boldsymbol{V} \in \operatorname{Span}\left(\boldsymbol{V}_{k}\right)_{0 \leq k \leq 2 n+2}, \mathrm{D}^{2} J\left(\omega^{*}\right) \cdot(\boldsymbol{V}, \boldsymbol{V}) \geq C_{n}|\boldsymbol{V}|^{2},
$$

where $C_{n}$ is a positive constant. But this constant $C_{n}$ tends to 0 when $n$ tends to $+\infty$. We even expect that this decreasing is exponential as observed in Ref. 2 for the Laplacian case. Therefore, our functional is degenerate for the highly oscillating deformations, i.e. for the deformation directions $\boldsymbol{V}_{k}$ with $k>>1$. Thus, for a numerical resolution, one has to exclude these highly oscillating deformations. We therefore explain the result of Conca et al. in Ref. 12. This instability can be avoided by regularization, for example, by adding to the least-squares functional a penalization in terms of the perimeter. This term leads to well posed problems (see Ref. 10 and Ref. 13). This lack of real stability is also pointed out in the recent paper of Ballerini (Ref. 8).

\section{Differentiability results}

Existence and characterization of the shape derivatives of the state and of the gradient of the least-squares functional are proved by classical arguments in shape calculus. Differentiability of the solution of the Stokes problem and of the shape functional is obtained through a generalized implicit function theorem proved by Simon (see Theorem 6 in Ref. 23) that we recall the statement for the reader's convenience.

All the proofs are done for the Neumann case. Adaption to the Dirichlet case is straightforward. Auxiliary results on Stokes equations with Neumann conditions (an existence and uniqueness of the solution theorem and a local regularity result) are recalled in Appendix A. 
Theorem 3.1 (J. Simon, Ref. 23). We give us

- an open set $\mathcal{U}$ in a Banach space $U, u_{0} \in \mathcal{U}$, two reflexive Banach spaces $E_{1}$ and $E_{2}$,

- a map $F: \mathcal{U} \times E_{1} \rightarrow E_{2}$, such that $F(u, \cdot) \in \mathcal{L}\left(E_{1}, E_{2}\right)$ for all $u \in \mathcal{U}$,

- a function $m: \mathcal{U} \rightarrow E_{1}$ and a function $f: \mathcal{U} \rightarrow E_{2}$ such that

$$
F(u, m(u))=f(u) \forall u \in \mathcal{U} .
$$

(i) Assume that

- $u \mapsto F(u, \cdot)$ is differentiable at $u_{0}$ into $\mathcal{L}\left(E_{1}, E_{2}\right)$,

- $f$ is differentiable at $u_{0}$,

- $\left\|F\left(u_{0}, x\right)\right\|_{E_{2}} \geq \alpha\|x\|_{E_{1}} \forall x \in E_{1}$, for some $\alpha>0$.

Then, the map $u \mapsto m(u)$ is differentiable at $u_{0}$. Its derivative $m^{\prime}\left(u_{0}, \cdot\right)$ is the unique solution of

$$
F\left(u_{0}, m^{\prime}\left(u_{0}, v\right)\right)=f^{\prime}\left(u_{0}, v\right)-\partial_{u} F\left(u_{0}, m\left(u_{0}\right), v\right) \quad \forall v \in U .
$$

(ii) In addition, assume that for some integer $k \geq 1, u \mapsto F(u, \cdot)$ and $f$ are $k$ times differentiable at $u_{0}$. Then, the map $u \mapsto m(u)$ is $k$ times differentiable at $u_{0}$.

We recall the main assumptions made in the introduction: $\boldsymbol{f} \in \mathbf{H}^{3}\left(\mathbb{R}^{N}\right), \Omega$ is Lipschitz and $\omega$ has a $C^{2,1}$ boundary. Let $\boldsymbol{\theta} \in \mathcal{U}$. We set $\left(\boldsymbol{u}_{\boldsymbol{\theta}}, p_{\theta}\right)$ the unique solution in $\mathbf{H}^{1}\left(\Omega \backslash \overline{\omega_{\theta}}\right) \times \mathrm{L}^{2}\left(\Omega \backslash \overline{\omega_{\theta}}\right)$ of

$$
\left\{\begin{aligned}
-\nu \Delta \boldsymbol{u}_{\boldsymbol{\theta}}+\nabla p_{\theta} & =\boldsymbol{f} \text { in } \Omega \backslash \overline{\omega_{\theta}} \\
\operatorname{div} \boldsymbol{u}_{\boldsymbol{\theta}} & =0 \text { in } \Omega \backslash \overline{\omega_{\theta}} \\
-\nu \partial_{\mathbf{n}} \boldsymbol{u}_{\boldsymbol{\theta}}+p_{\theta} \mathbf{n} & =\mathbf{0} \text { on } \partial \Omega \\
-\nu \partial_{\mathbf{n}_{\boldsymbol{\theta}}} \boldsymbol{u}_{\boldsymbol{\theta}}+p_{\theta} \mathbf{n}_{\boldsymbol{\theta}} & =\mathbf{0} \text { on } \partial \omega_{\theta}
\end{aligned}\right.
$$

with

$$
\int_{O} u_{\theta}=\int_{O} f_{b}
$$

This boundary values problem has the following variational formulation:

$$
\left\{\begin{aligned}
\int_{\Omega \backslash \overline{\omega_{\theta}}}\left\{\nu \nabla \boldsymbol{u}_{\boldsymbol{\theta}}: \nabla \boldsymbol{\varphi}_{\boldsymbol{\theta}}-p_{\theta} \operatorname{div} \boldsymbol{\varphi}_{\boldsymbol{\theta}}\right\} & =\int_{\Omega \backslash \overline{\omega_{\theta}}} \boldsymbol{f} \cdot \boldsymbol{\varphi}_{\boldsymbol{\theta}} \forall \boldsymbol{\varphi}_{\boldsymbol{\theta}} \in \mathbf{H}^{1}\left(\Omega \backslash \overline{\omega_{\theta}}\right), \\
\int_{\Omega \backslash \overline{\omega_{\theta}}} \xi_{\theta} \operatorname{div} \boldsymbol{u}_{\boldsymbol{\theta}} & =0 \quad \forall \xi_{\theta} \in \mathrm{L}^{2}\left(\Omega \backslash \overline{\bar{\omega}_{\theta}}\right), \\
\int_{O} \boldsymbol{u}_{\boldsymbol{\theta}} & =\int_{O} \boldsymbol{f}_{\boldsymbol{b}} .
\end{aligned}\right.
$$

Let us define the key objects of our differentiability proof:

$$
\boldsymbol{v}_{\boldsymbol{\theta}}:=\boldsymbol{u}_{\boldsymbol{\theta}} \circ(\mathbf{I}+\boldsymbol{\theta}) \in \mathbf{H}^{1}(\Omega \backslash \bar{\omega}) \quad \text { and } \quad q_{\theta}:=p_{\theta} \circ(\mathbf{I}+\boldsymbol{\theta}) \in \mathrm{L}^{2}(\Omega \backslash \bar{\omega}) .
$$


For $k, m \in \mathbb{N}, k<m$, we note $\mathrm{X}^{k, m}\left(\Omega \backslash \bar{\omega}, \Omega_{\delta} \backslash \bar{\omega}\right)$ the space of functions in $\mathrm{H}^{k}(\Omega \backslash \bar{\omega})$ such that their restriction to $\Omega_{\delta} \backslash \bar{\omega}$ belongs to $\mathrm{H}^{m}\left(\Omega_{\delta} \backslash \bar{\omega}\right)$. This space endowed with the norm

$$
\|u\|_{\mathrm{X}^{k, m}\left(\Omega \backslash \bar{\omega}, \Omega_{\delta} \backslash \bar{\omega}\right)}:=\left(\|u\|_{\mathrm{H}^{k}(\Omega \backslash \bar{\omega})}^{2}+\|u\|_{\mathrm{H}^{m}\left(\Omega_{\delta} \backslash \bar{\omega}\right)}^{2}\right)^{1 / 2},
$$

is hilbertian. We define similarly the space $\mathrm{X}^{*, m}\left(\Omega \backslash \bar{\omega}, \Omega_{\delta} \backslash \bar{\omega}\right)$ the space of functions in $\left[\mathrm{H}^{1}(\Omega \backslash \bar{\omega})\right]^{\prime}$ such that their restriction to $\Omega_{\delta} \backslash \bar{\omega}$ belongs to $\mathrm{H}^{m}\left(\Omega_{\delta} \backslash \bar{\omega}\right)$.

Before establishing the first order differentiability, we prove the following three lemmas.

Lemma 3.1 (Characterization of $\left(\boldsymbol{v}_{\boldsymbol{\theta}}, q_{\theta}\right)$ ). For $\boldsymbol{\theta} \in \mathcal{U}$, the pair $\left(\boldsymbol{v}_{\boldsymbol{\theta}}, q_{\theta}\right)$ satisfies for all $\varphi \in \mathbf{H}^{1}(\Omega \backslash \bar{\omega})$ and all $\xi \in \mathrm{L}^{2}(\Omega \backslash \bar{\omega})$

$$
\left\{\begin{aligned}
\int_{\Omega \backslash \bar{\omega}}\left\{\left[\nabla \boldsymbol{v}_{\boldsymbol{\theta}} A(\boldsymbol{\theta})\right]: \nabla \boldsymbol{\varphi}-q_{\theta} B(\boldsymbol{\theta}): \nabla \boldsymbol{\varphi}\right\} & =\int_{\Omega \backslash \bar{\omega}}[\boldsymbol{f} \circ(\mathbf{I}+\boldsymbol{\theta})] \cdot \boldsymbol{\varphi} J_{\boldsymbol{\theta}} \\
\int_{\Omega \backslash \bar{\omega}}\left(\nabla \boldsymbol{v}_{\boldsymbol{\theta}}: B(\boldsymbol{\theta})\right) \xi & =0 \\
\int_{O} \boldsymbol{v}_{\boldsymbol{\theta}} & =\int_{O} \boldsymbol{f}_{\boldsymbol{b}}
\end{aligned}\right.
$$

with

$$
\begin{aligned}
J_{\boldsymbol{\theta}} & :=\operatorname{det}(\mathrm{I}+\nabla \boldsymbol{\theta}) \in \mathrm{W}^{1, \infty}\left(\overline{\Omega_{\delta}}\right), \\
A(\boldsymbol{\theta}) & :=\nu J_{\boldsymbol{\theta}}(\mathrm{I}+\nabla \boldsymbol{\theta})^{-1}\left(\mathrm{I}+{ }^{t} \nabla \boldsymbol{\theta}\right)^{-1} \in \mathrm{W}^{1, \infty}\left(\overline{\Omega_{\delta}}, \mathcal{M}_{N, N}\right), \\
B(\boldsymbol{\theta}) & :=J_{\boldsymbol{\theta}}\left(\mathrm{I}+{ }^{t} \nabla \boldsymbol{\theta}\right)^{-1} \in \mathrm{W}^{1, \infty}\left(\overline{\Omega_{\delta}}, \mathcal{M}_{N, N}\right) .
\end{aligned}
$$

Lemma 3.2 (Differentiability of $\boldsymbol{\theta} \mapsto\left(\boldsymbol{v}_{\boldsymbol{\theta}}, q_{\theta}\right)$ ). The function

$$
\boldsymbol{\theta} \in \mathcal{U} \mapsto\left(\boldsymbol{v}_{\boldsymbol{\theta}}, q_{\theta}\right) \in \mathbf{X}^{1,2}\left(\Omega \backslash \bar{\omega}, \Omega_{\delta} \backslash \bar{\omega}\right) \times \mathrm{X}^{0,1}\left(\Omega \backslash \bar{\omega}, \Omega_{\delta} \backslash \bar{\omega}\right)
$$

is differentiable in a neighborhood of $\mathbf{0}$.

Lemma 3.3 (Differentiability of $\boldsymbol{\theta} \mapsto\left(\boldsymbol{u}_{\boldsymbol{\theta}}, p_{\theta}\right)$ ). There exists $\widetilde{\boldsymbol{u}}_{\boldsymbol{\theta}}, \widetilde{p}_{\theta}$ some respective extension of $\boldsymbol{u}_{\boldsymbol{\theta}} \in \mathbf{H}^{1}(\Omega \backslash \bar{\omega}), p_{\theta} \in \mathrm{L}^{2}(\Omega \backslash \bar{\omega})$ such that the functions

$$
\boldsymbol{\theta} \in \mathcal{U} \mapsto \widetilde{\boldsymbol{u}}_{\boldsymbol{\theta}} \in \mathbf{H}^{1}(\Omega) \quad \text { and } \quad \boldsymbol{\theta} \in \mathcal{U} \mapsto \widetilde{p}_{\theta} \in \mathrm{L}^{2}(\Omega)
$$

are differentiable at $\mathbf{0 .}$

Remark 3.1. We will prove this three lemmas under weaker geometrical assumptions: $\boldsymbol{f} \in \mathbf{H}^{1}\left(\mathbb{R}^{N}\right)$ and $\omega$ with a $C^{1,1}$ boundary.

Proof. (of Lemma 3.1: characterization of $\left(\boldsymbol{v}_{\boldsymbol{\theta}}, q_{\theta}\right)$ ). Let $\varphi \in \mathbf{H}^{1}(\Omega \backslash \bar{\omega})$, $\xi \in \mathrm{L}^{2}(\Omega \backslash \bar{\omega})$ and $\boldsymbol{\theta} \in \mathcal{U}$. Using

$$
\boldsymbol{\varphi}_{\boldsymbol{\theta}}:=\boldsymbol{\varphi} \circ(\mathbf{I}+\boldsymbol{\theta})^{-1} \in \mathbf{H}^{1}\left(\Omega \backslash \overline{\omega_{\theta}}\right) \quad \text { and } \quad \xi_{\theta}:=\xi \circ(\mathbf{I}+\boldsymbol{\theta})^{-1} \in \mathrm{L}^{2}\left(\Omega \backslash \overline{\omega_{\theta}}\right)
$$


as test functions in the variational formulation (3.2), we have

$$
\left\{\begin{array}{c}
\int_{\Omega \backslash \overline{\omega_{\theta}}}\left\{\nu\left[\left(\nabla \boldsymbol{v}_{\boldsymbol{\theta}}(\mathrm{I}+\nabla \boldsymbol{\theta})^{-1}\right) \circ(\mathbf{I}+\boldsymbol{\theta})^{-1}\right]:\left[\left(\nabla \boldsymbol{\varphi}(\mathrm{I}+\nabla \boldsymbol{\theta})^{-1}\right) \circ(\mathbf{I}+\boldsymbol{\theta})^{-1}\right]\right. \\
\left.-\left(q_{\theta} \circ(\mathbf{I}+\boldsymbol{\theta})^{-1}\right) \operatorname{div}\left(\boldsymbol{\varphi} \circ(\mathbf{I}+\boldsymbol{\theta})^{-1}\right)\right\}=\int_{\Omega \backslash \overline{\omega_{\theta}}} \boldsymbol{f} \cdot\left[\boldsymbol{\varphi} \circ(\mathbf{I}+\boldsymbol{\theta})^{-1}\right], \\
\int_{\Omega \backslash \overline{\omega_{\theta}}}\left[\xi \circ(\mathbf{I}+\boldsymbol{\theta})^{-1}\right] \operatorname{div}\left(\boldsymbol{v}_{\boldsymbol{\theta}} \circ(\mathbf{I}+\boldsymbol{\theta})^{-1}\right)=0, \\
\int_{O} \boldsymbol{v}_{\boldsymbol{\theta}} \circ(\mathbf{I}+\boldsymbol{\theta})^{-1}=\int_{O} \boldsymbol{f}_{\boldsymbol{b}} .
\end{array}\right.
$$

However, $\operatorname{div}\left(\boldsymbol{\varphi} \circ(\mathbf{I}+\boldsymbol{\theta})^{-1}\right)=\left(\nabla \boldsymbol{\varphi} \circ(\mathbf{I}+\boldsymbol{\theta})^{-1}\right):\left(\left(\mathrm{I}+{ }^{t} \nabla \boldsymbol{\theta}\right)^{-1} \circ(\mathbf{I}+\boldsymbol{\theta})^{-1}\right)$, and a similar equality holds with $\boldsymbol{v}_{\boldsymbol{\theta}}$ instead of $\boldsymbol{\varphi}$. Thus, the conclusion follows after the change of variables $x=(\mathbf{I}+\boldsymbol{\theta}) y$ and by noticing that

$$
\int_{O} \boldsymbol{v}_{\boldsymbol{\theta}} J_{\boldsymbol{\theta}}=\int_{O}\left[\boldsymbol{f}_{\boldsymbol{b}} \circ(\mathbf{I}+\boldsymbol{\theta})\right] J_{\boldsymbol{\theta}} \Longleftrightarrow \int_{O} \boldsymbol{v}_{\boldsymbol{\theta}}=\int_{O} \boldsymbol{f}_{\boldsymbol{b}},
$$

since $\boldsymbol{\theta} \equiv \mathbf{0}$ on $O \subset \partial \Omega$.

Proof. (of Lemma 3.2: differentiability of $\theta \mapsto\left(\boldsymbol{v}_{\boldsymbol{\theta}}, q_{\theta}\right)$ ). Let us check the assumptions of Simon's Theorem.

First step: notations. We need some additional tools: a third domain $\widetilde{\Omega}_{\delta}$ which is an open set with a $C^{\infty}$ boundary and such that $\Omega_{\delta} \subset \subset \widetilde{\Omega}_{\delta} \subset \subset \Omega$ and a truncation function $\Phi \in C_{c}^{\infty}\left(\widetilde{\Omega}_{\delta}\right)$ such that $\Phi \equiv 1$ in $\Omega_{\delta}$. Using the notations introduced in Lemma 3.1, we define the spaces:

$$
\begin{aligned}
& \boldsymbol{E}_{\mathbf{1}}:=\left\{(\boldsymbol{v}, q) \in \mathbf{H}^{1}(\Omega \backslash \bar{\omega}) \times \mathrm{L}^{2}(\Omega \backslash \bar{\omega}) ;(\Phi \boldsymbol{v}, \Phi q) \in \mathbf{H}^{2}(\Omega \backslash \bar{\omega}) \times \mathrm{H}^{1}(\Omega \backslash \bar{\omega})\right\}, \\
& \boldsymbol{E}_{\mathbf{2}}:=\left\{(\boldsymbol{f}, g) \in\left[\mathbf{H}^{1}(\Omega \backslash \bar{\omega})\right]^{\prime} \times \mathrm{L}^{2}(\Omega \backslash \bar{\omega}) ;(\Phi \boldsymbol{f}, \Phi g) \in \mathbf{L}^{2}(\Omega \backslash \bar{\omega}) \times \mathrm{H}^{1}(\Omega \backslash \bar{\omega})\right\} \times \mathbb{R}^{N} .
\end{aligned}
$$

Note that $\boldsymbol{E}_{\mathbf{1}}$ and $\boldsymbol{E}_{\mathbf{2}}$ are Hilbert spaces with respective norms

$$
\begin{gathered}
\|(\boldsymbol{v}, q)\|_{\boldsymbol{E}_{\mathbf{1}}}^{2}:=\|\boldsymbol{v}\|_{\mathbf{H}^{1}(\Omega \backslash \bar{\omega})}^{2}+\|q\|_{\mathrm{L}^{2}(\Omega \backslash \bar{\omega})}^{2}+\|\Phi \boldsymbol{v}\|_{\mathbf{H}^{2}(\Omega \backslash \bar{\omega})}^{2}+\|\Phi q\|_{\mathrm{H}^{1}(\Omega \backslash \bar{\omega})}^{2}, \\
\|((\boldsymbol{f}, g), \boldsymbol{r})\|_{\boldsymbol{E}_{\mathbf{2}}}^{2}:=\|\boldsymbol{f}\|_{\left[\mathbf{H}^{1}(\Omega \backslash \bar{\omega})\right]^{\prime}}^{2}+\|g\|_{\mathrm{L}^{2}(\Omega \backslash \bar{\omega})}^{2}+\|\Phi \boldsymbol{f}\|_{\mathbf{L}^{2}(\Omega \backslash \bar{\omega})}^{2}+\|\Phi g\|_{\mathrm{H}^{1}(\Omega \backslash \bar{\omega})}^{2}+|\boldsymbol{r}|^{2} .
\end{gathered}
$$

Moreover, for $(\boldsymbol{v}, q) \in \boldsymbol{E}_{\mathbf{1}},(\boldsymbol{v}, q) \in \mathbf{X}^{1,2}\left(\Omega \backslash \bar{\omega}, \Omega_{\delta} \backslash \bar{\omega}\right) \times \mathrm{X}^{0,1}\left(\Omega \backslash \bar{\omega}, \Omega_{\delta} \backslash \bar{\omega}\right)$, and for $((\boldsymbol{f}, g), \boldsymbol{r}) \in \boldsymbol{E}_{\mathbf{2}},(\boldsymbol{f}, g) \in \mathbf{X}^{*, 0}\left(\Omega \backslash \bar{\omega}, \Omega_{\delta} \backslash \bar{\omega}\right) \times \mathrm{X}^{0,1}\left(\Omega \backslash \bar{\omega}, \Omega_{\delta} \backslash \bar{\omega}\right)$, and there is a constant $C_{E}$ such that the following estimates hold:

$$
\begin{aligned}
&\|(\boldsymbol{v}, q)\|_{\mathbf{X}^{1,2}\left(\Omega \backslash \bar{\omega}, \Omega_{\delta} \backslash \bar{\omega}\right) \times \mathrm{X}^{0,1}\left(\Omega \backslash \bar{\omega}, \Omega_{\delta} \backslash \bar{\omega}\right)} \leq C_{E}\|(\boldsymbol{v}, q)\|_{\boldsymbol{E}_{\mathbf{1}}} \\
&\|(\boldsymbol{f}, g)\|_{\mathbf{X}^{*, 0}\left(\Omega \backslash \bar{\omega}, \Omega_{\delta} \backslash \bar{\omega}\right) \times \mathrm{X}^{0,1}\left(\Omega \backslash \bar{\omega}, \Omega_{\delta} \backslash \bar{\omega}\right)} \leq C_{E}\|((\boldsymbol{f}, g), \boldsymbol{r})\|_{\boldsymbol{E}_{\mathbf{2}}} .
\end{aligned}
$$

We also define the functions:

- $\boldsymbol{f}_{\mathbf{1}}(\boldsymbol{\theta},(\boldsymbol{v}, q)) \in\left[\mathbf{H}^{1}(\Omega \backslash \bar{\omega})\right]^{\prime}$ by $\forall \boldsymbol{\varphi} \in \mathbf{H}^{1}(\Omega \backslash \bar{\omega})$,

$$
\left\langle\boldsymbol{f}_{\mathbf{1}}(\boldsymbol{\theta},(\boldsymbol{v}, q)), \boldsymbol{\varphi}\right\rangle_{\Omega \backslash \bar{\omega}}:=\int_{\Omega \backslash \bar{\omega}}[\boldsymbol{f} \circ(\mathbf{I}+\boldsymbol{\theta})] \cdot J_{\boldsymbol{\theta}} \boldsymbol{\varphi}
$$


- $\boldsymbol{F}_{\mathbf{1}}(\boldsymbol{\theta},(\boldsymbol{v}, q)) \in\left[\mathbf{H}^{1}(\Omega \backslash \bar{\omega})\right]^{\prime}$ by $\forall \boldsymbol{\varphi} \in \mathbf{H}^{1}(\Omega \backslash \bar{\omega})$,

$$
\left\langle\boldsymbol{F}_{\mathbf{1}}(\boldsymbol{\theta},(\boldsymbol{v}, q)), \boldsymbol{\varphi}\right\rangle_{\Omega \backslash \bar{\omega}}:=\int_{\Omega \backslash \bar{\omega}}\{[\nabla \boldsymbol{v} A(\boldsymbol{\theta})]: \nabla \boldsymbol{\varphi}-q B(\boldsymbol{\theta}): \nabla \boldsymbol{\varphi}\},
$$

- $\boldsymbol{m}(\boldsymbol{\theta}):=\left(\boldsymbol{v}_{\boldsymbol{\theta}}, q_{\theta}\right) \quad$ and $\quad \boldsymbol{f}(\boldsymbol{\theta}):=\left(\boldsymbol{f}_{\mathbf{1}}(\boldsymbol{\theta},(\boldsymbol{v}, q)), 0, \int_{O} \boldsymbol{f}_{\boldsymbol{b}}\right)$,

- $\boldsymbol{F}(\boldsymbol{\theta},(\boldsymbol{v}, q)):=\left(\boldsymbol{F}_{\mathbf{1}}(\boldsymbol{\theta},(\boldsymbol{v}, q)), \nabla \boldsymbol{v}: B(\boldsymbol{\theta}), \int_{O} \boldsymbol{v}\right)$.

By the characterization of $\left(\boldsymbol{v}_{\boldsymbol{\theta}}, q_{\theta}\right)$ obtained in Lemma 3.1,

$$
\boldsymbol{F}(\boldsymbol{\theta}, \boldsymbol{m}(\boldsymbol{\theta}))=\boldsymbol{f}(\boldsymbol{\theta}) \quad \forall \boldsymbol{\theta} \in \mathcal{U} .
$$

Second step: differentiability of $\boldsymbol{F}$ and $\boldsymbol{f}$ at $\mathbf{0}$. We use the chain rule. Since the functions

$$
\boldsymbol{\theta} \in \mathcal{U} \mapsto J_{\theta} \in \mathrm{L}^{\infty}(\bar{\Omega}) \quad \text { and } \quad \boldsymbol{\theta} \in \mathcal{U} \mapsto(\mathrm{I}+\nabla \boldsymbol{\theta})^{-1} \in \mathrm{L}^{\infty}\left(\bar{\Omega}, \mathcal{M}_{N \times N}\right)
$$

are $C^{\infty}$, the functions

$$
\boldsymbol{\theta} \in \mathcal{U} \mapsto A(\boldsymbol{\theta}) \in \mathrm{L}^{\infty}\left(\bar{\Omega}, \mathcal{M}_{N \times N}\right) \quad \text { and } \quad \boldsymbol{\theta} \in \mathcal{U} \mapsto B(\boldsymbol{\theta}) \in \mathrm{L}^{\infty}\left(\bar{\Omega}, \mathcal{M}_{N \times N}\right)
$$

are $C^{\infty}$. Moreover, the following functions are $C^{\infty}$ :

$\mathrm{L}^{\infty}\left(\bar{\Omega}, \mathcal{M}_{N \times N}\right) \times\left\{\boldsymbol{v} \in \mathbf{H}^{1}(\Omega \backslash \bar{\omega}) ; \Phi \boldsymbol{v} \in \mathbf{H}^{2}(\Omega \backslash \bar{\omega})\right\} \rightarrow\left\{g \in \mathrm{L}^{2}(\Omega \backslash \bar{\omega}) ; \Phi g \in \mathrm{H}^{1}(\Omega \backslash \bar{\omega})\right\}$ $(B, \boldsymbol{v}) \mapsto \nabla \boldsymbol{v}: B$,

$$
\boldsymbol{v} \in \mathbf{X}^{1,2}\left(\Omega \backslash \bar{\omega}, \Omega_{\delta} \backslash \bar{\omega}\right) \mapsto \int_{O} \boldsymbol{v} \in \mathbb{R}^{N},
$$

$\boldsymbol{F}_{\mathbf{1}}:(\boldsymbol{\theta},(\boldsymbol{v}, q)) \in \mathcal{U} \times \boldsymbol{E}_{\mathbf{1}} \mapsto \boldsymbol{F}_{\mathbf{1}}(\boldsymbol{\theta},(\boldsymbol{v}, q)) \in\left\{\boldsymbol{f} \in\left[\mathbf{H}^{1}(\Omega \backslash \bar{\omega})\right]^{\prime} ; \Phi \boldsymbol{f} \in \mathbf{L}^{2}(\Omega \backslash \bar{\omega})\right\}$.

Finally, since $\boldsymbol{f} \in \mathbf{H}^{1}\left(\mathbb{R}^{N}\right)$ by hypothesis, the function

$$
\boldsymbol{\theta} \in \mathbf{W}^{1, \infty}(\bar{\Omega}) \mapsto \boldsymbol{f} \circ(\mathbf{I}+\boldsymbol{\theta}) \in \mathbf{L}^{2}\left(\mathbb{R}^{N}\right)
$$

is $C^{1}$ in a neighborhood of $\mathbf{0}$ by Lemma 5.3.3 in Ref. 20. Then, the function

$$
\boldsymbol{f}_{\mathbf{1}}:(\boldsymbol{\theta},(\boldsymbol{v}, q)) \in \mathcal{U} \times \boldsymbol{E}_{\mathbf{1}} \mapsto \boldsymbol{f}_{\mathbf{1}}(\boldsymbol{\theta},(\boldsymbol{v}, q)) \in\left\{\boldsymbol{f} \in\left[\mathbf{H}^{1}(\Omega \backslash \bar{\omega})\right]^{\prime} ; \Phi \boldsymbol{f} \in \mathbf{L}^{2}(\Omega \backslash \bar{\omega})\right\}
$$

is $C^{1}$. By composition, $\boldsymbol{F}$ is $C^{\infty}$ and $\boldsymbol{f}$ is $C^{1}$ in a neighborhood of $\mathbf{0}$.

Third step: existence of $\alpha>0$ such that $\|\boldsymbol{F}(\mathbf{0},(\boldsymbol{v}, q))\|_{\boldsymbol{E}_{2}} \geq \alpha\|(\boldsymbol{v}, q)\|_{\boldsymbol{E}_{1}}$. We consider a pair $(\boldsymbol{v}, q) \in \boldsymbol{E}_{\mathbf{1}}$ and we define $(\boldsymbol{\xi}, \eta, \boldsymbol{r}) \in \boldsymbol{E}_{\mathbf{2}}$ by $\boldsymbol{F}(\mathbf{0},(\boldsymbol{v}, q))=(\boldsymbol{\xi}, \eta, \boldsymbol{r})$. Then, we have, for all $\varphi \in \mathbf{H}^{1}(\Omega \backslash \bar{\omega})$,

$$
\left\{\begin{aligned}
\int_{\Omega \backslash \bar{\omega}}\{\nu \nabla \boldsymbol{v}: \nabla \boldsymbol{\varphi}-q \operatorname{div} \boldsymbol{\varphi}\} & =\langle\boldsymbol{\xi}, \boldsymbol{\varphi}\rangle_{\Omega \backslash \bar{\omega}}, \\
\operatorname{div} \boldsymbol{v} & =\eta \\
\int_{O} \boldsymbol{v} & =\boldsymbol{r} .
\end{aligned}\right.
$$


Setting $\varphi_{i}:={ }^{t}(0, \ldots, 0,1,0, \ldots, 0),(i=1, \ldots, N)$, as test functions in the first line of the previous system, we check the compatibility condition of Appendix A.1. Thus, according to Appendix A.1, there exists $\boldsymbol{w} \in \mathbf{H}^{1}(\Omega \backslash \bar{\omega})$ such that $\int_{\Omega \backslash \bar{\omega}} \boldsymbol{w}=\mathbf{0}$ and such that, for all $\varphi \in \mathbf{H}^{1}(\Omega \backslash \bar{\omega})$,

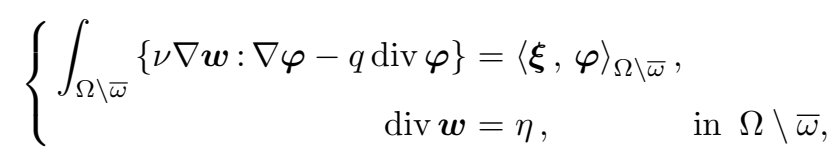

and there exits a constant $\alpha_{1}>0$ such that

$$
\|\boldsymbol{w}\|_{\mathbf{H}^{1}(\Omega \backslash \bar{\omega})}+\|q\|_{L^{2}(\Omega \backslash \bar{\omega})} \leq \alpha_{1}\left(\|\boldsymbol{\xi}\|_{\left[\mathbf{H}^{1}(\Omega \backslash \bar{\omega})\right]^{\prime}}+\|\eta\|_{L^{2}(\Omega \backslash \bar{\omega})}\right) .
$$

Such a problem has a unique solution, we then get that

$$
\boldsymbol{v}=\boldsymbol{w}-\frac{1}{|O|} \int_{O} \boldsymbol{w}+\boldsymbol{r}
$$

Thus, by computation, there exists a constant $\alpha_{2}>0$ such that

$$
\|\boldsymbol{v}\|_{\mathbf{H}^{1}(\Omega \backslash \bar{\omega})}-|\Omega \backslash \bar{\omega}|^{-1 / 2}|\boldsymbol{r}| \leq \alpha_{2}\|\boldsymbol{w}\|_{\mathbf{H}^{1}(\Omega \backslash \bar{\omega})} .
$$

Hence, using the above inequality and (3.4), there exists a constant $\alpha_{3}>0$, such that

$$
\|\boldsymbol{v}\|_{\mathbf{H}^{1}(\Omega \backslash \bar{\omega})}+\|q\|_{L^{2}(\Omega \backslash \bar{\omega})} \leq \alpha_{3}\left(\|\boldsymbol{\xi}\|_{\left[\mathbf{H}^{1}(\Omega \backslash \bar{\omega})\right]^{\prime}}+\|\eta\|_{L^{2}(\Omega \backslash \bar{\omega})}+|\boldsymbol{r}|\right) .
$$

Mimicking the proof of the local regularity result Appendix A.2 (see inequality (A.9)), we use the argument stated above to prove the existence of a constant $\alpha_{4}$ such that

$$
\begin{aligned}
& \|\Phi \boldsymbol{v}\|_{\mathbf{H}^{2}\left(\widetilde{\Omega}_{\delta} \backslash \bar{\omega}\right)}+\|\Phi q\|_{\mathrm{H}^{1}\left(\widetilde{\Omega}_{\delta} \backslash \bar{\omega}\right)} \\
& \quad \leq \alpha_{4}\left(\|\Phi \boldsymbol{\xi}\|_{\mathbf{L}^{2}\left(\widetilde{\Omega}_{\delta} \backslash \bar{\omega}\right)}+\|\Phi \eta\|_{\mathrm{H}^{1}\left(\widetilde{\Omega}_{\delta} \backslash \bar{\omega}\right)}+\|\boldsymbol{v}\|_{\mathbf{H}^{1}(\Omega \backslash \bar{\omega})}+\|q\|_{\mathrm{L}^{2}(\Omega \backslash \bar{\omega})}+|\boldsymbol{r}|\right) .
\end{aligned}
$$

Thus, since $\Phi$ is compactly supported in $\widetilde{\Omega}_{\delta}$,

$$
\begin{aligned}
& \|\Phi \boldsymbol{v}\|_{\mathbf{H}^{2}(\Omega \backslash \bar{\omega})}+\|\Phi q\|_{\mathrm{H}^{1}(\Omega \backslash \bar{\omega})} \\
& \quad \leq \alpha_{4}\left(\|\Phi \boldsymbol{\xi}\|_{\mathbf{L}^{2}(\Omega \backslash \bar{\omega})}+\|\Phi \eta\|_{\mathrm{H}^{1}(\Omega \backslash \bar{\omega})}+\|\boldsymbol{v}\|_{\mathbf{H}^{1}(\Omega \backslash \bar{\omega})}+\|q\|_{\mathrm{L}^{2}(\Omega \backslash \bar{\omega})}+|\boldsymbol{r}|\right) .
\end{aligned}
$$

Gathering (3.5) and (3.6), there exists a constant $\alpha>0$ such that

$$
\|\boldsymbol{F}(\mathbf{0},(\boldsymbol{v}, q))\|_{\boldsymbol{E}_{\mathbf{2}}} \geq \alpha\|(\boldsymbol{v}, q)\|_{\boldsymbol{E}_{\mathbf{1}}} .
$$

Fourth step: conclusion. By Simon's Theorem,

$$
\boldsymbol{\theta} \in \mathcal{U} \mapsto\left(\boldsymbol{v}_{\boldsymbol{\theta}}, q_{\theta}\right) \in \boldsymbol{E}_{\mathbf{1}}
$$

is differentiable in a neighborhood of $\mathbf{0}$. We conclude using the fact that $\boldsymbol{E}_{\mathbf{1}}$ is continuously embedded in $\mathbf{X}^{1,2}\left(\Omega \backslash \bar{\omega}, \Omega_{\delta} \backslash \bar{\omega}\right) \times \mathrm{X}^{0,1}\left(\Omega \backslash \bar{\omega}, \Omega_{\delta} \backslash \bar{\omega}\right)$ (see (3.3)). 
Proof. (of Lemma 3.3: differentiability of $\boldsymbol{\theta} \mapsto\left(\boldsymbol{u}_{\boldsymbol{\theta}}, p_{\theta}\right)$ ). We use the chain rule. We only prove the result on $\widetilde{\boldsymbol{u}}_{\boldsymbol{\theta}}$ because the ideas are exactly the same for the result on $\widetilde{p}_{\theta}$.

For $\boldsymbol{\theta} \in \mathcal{U}, \boldsymbol{u}_{\boldsymbol{\theta}}=\boldsymbol{v}_{\boldsymbol{\theta}} \circ(\mathbf{I}+\boldsymbol{\theta})^{-1} \in \mathbf{H}^{2}\left(\Omega_{\delta} \backslash \overline{\omega_{\theta}}\right)$. According to Lemma 3.2 and Stein's extension Theorem (Theorem 5.24 in Ref. 1), there exists $\widetilde{\boldsymbol{v}}_{\boldsymbol{\theta}}$, an extension of $\boldsymbol{v}_{\boldsymbol{\theta}}$, such that $\boldsymbol{\theta} \in \mathcal{U} \mapsto \widetilde{\boldsymbol{v}}_{\boldsymbol{\theta}} \in \mathbf{H}^{2}\left(\Omega_{\delta}\right)$ is differentiable at $\mathbf{0}$. Moreover,

$$
\boldsymbol{\theta} \in \mathcal{U} \mapsto(\mathbf{I}+\boldsymbol{\theta})^{-1}-\mathbf{I} \in \mathbf{W}^{1, \infty}(\bar{\Omega})
$$

is differentiable at $\mathbf{0}$. Thus,

$$
\boldsymbol{\varphi}_{\mathbf{1}}: \boldsymbol{\theta} \in \mathcal{U} \mapsto\left(\widetilde{\boldsymbol{v}}_{\boldsymbol{\theta}},(\mathbf{I}+\boldsymbol{\theta})^{-1}-\mathbf{I}\right) \in \mathbf{H}^{2}\left(\Omega_{\delta}\right) \times \mathbf{W}^{1, \infty}(\bar{\Omega})
$$

is differentiable at $\mathbf{0}$. We apply Lemma 5.3.9 in Ref. 20, to get that

$$
\varphi_{2}:(\boldsymbol{g}, \boldsymbol{\mu}) \in \mathbf{H}^{2}\left(\mathbb{R}^{N}\right) \times \mathbf{W}^{1, \infty}(\bar{\Omega}) \mapsto \boldsymbol{g} \circ(\mathbf{I}+\boldsymbol{\mu}) \in \mathbf{H}^{1}\left(\mathbb{R}^{N}\right)
$$

is $C^{1}$ in a neighborhood of $\mathbf{0}$. By composition, $\varphi_{2} \circ \varphi_{1}$ is differentiable at $\mathbf{0}$. Then, we define an extension of $\boldsymbol{u}_{\boldsymbol{\theta}}$ as

$$
\widetilde{\boldsymbol{u}_{\boldsymbol{\theta}}}:=\left\{\begin{aligned}
\varphi_{2} \circ \varphi_{\mathbf{1}}(\boldsymbol{\theta}) & \text { in } \Omega_{\delta}, \\
\boldsymbol{u}_{\boldsymbol{\theta}} & \text { in } \Omega \backslash \overline{\Omega_{\delta}} .
\end{aligned}\right.
$$

Using Lemma 3.2, the function

$$
\boldsymbol{\theta} \in \mathcal{U} \mapsto \boldsymbol{u}_{\boldsymbol{\theta} \mid \Omega \backslash \overline{\Omega_{\delta}}}=\left.\boldsymbol{v}_{\boldsymbol{\theta}} \circ(\mathbf{I}+\boldsymbol{\theta})^{-1}\right|_{\Omega \backslash \overline{\Omega_{\delta}}}=\boldsymbol{v}_{\boldsymbol{\theta} \mid \Omega \backslash \overline{\Omega_{\delta}}} \in \mathbf{H}^{1}\left(\Omega \backslash \overline{\Omega_{\delta}}\right)
$$

is differentiable at $\mathbf{0}$. Moreover, on a neighborhood $\mathcal{V}\left(\partial \Omega_{\delta}\right)$ of $\partial \Omega_{\delta}, \widetilde{\widetilde{\boldsymbol{u}_{\boldsymbol{\theta}}}}=\boldsymbol{u}_{\boldsymbol{\theta}}=\boldsymbol{v}_{\boldsymbol{\theta}}$. Thus, using Lemma 3.2, $\boldsymbol{\theta} \in \mathcal{U} \mapsto \widetilde{\boldsymbol{u}_{\boldsymbol{\theta}}} \mid \mathcal{V}\left(\partial \Omega_{\delta}\right) \in \mathbf{H}^{1}\left(\mathcal{V}\left(\partial \Omega_{\delta}\right)\right)$ is differentiable at $\mathbf{0 .}$ Therefore $\boldsymbol{\theta} \in \mathcal{U} \mapsto \widetilde{\boldsymbol{u}_{\boldsymbol{\theta}}} \in \mathbf{H}^{1}(\Omega)$ is differentiable at $\mathbf{0}$.

Higher order differentiability. To prove that $(\boldsymbol{u}, p)$ is twice differentiable with respect to the shape, we use the second order differentiability of $\boldsymbol{\theta} \mapsto\left(\boldsymbol{v}_{\boldsymbol{\theta}}, q_{\theta}\right)$. Therefore, we first prove the following lemma:

Lemma 3.4. The function

$$
\boldsymbol{\theta} \in \mathcal{U} \mapsto\left(\boldsymbol{v}_{\boldsymbol{\theta}}, q_{\theta}\right) \in \mathbf{X}^{1,3}\left(\Omega \backslash \bar{\omega}, \Omega_{\delta} \backslash \bar{\omega}\right) \times \mathrm{X}^{0,2}\left(\Omega \backslash \bar{\omega}, \Omega_{\delta} \backslash \bar{\omega}\right)
$$

is twice differentiable in a neighborhood of $\mathbf{0}$.

Proof. We use Simon's Theorem 3.1. Let the open set $\widetilde{\Omega}_{\delta}$ defined in the proof of Lemma 3.2 and $\Phi \in C_{c}^{\infty}\left(\widetilde{\Omega}_{\delta}\right)$ such that $\Phi \equiv 1$ in $\Omega_{\delta}$. We consider the functions $\boldsymbol{F}$ and $\boldsymbol{f}$ defined in the proof of Lemma 3.2 with modified sets of departure and destination:

$$
\begin{aligned}
& \boldsymbol{E}_{\mathbf{1}}:=\left\{(\boldsymbol{v}, q) \in \mathbf{H}^{1}(\Omega \backslash \bar{\omega}) \times \mathrm{L}^{2}(\Omega \backslash \bar{\omega}) ;(\Phi \boldsymbol{v}, \Phi q) \in \mathbf{H}^{3}(\Omega \backslash \bar{\omega}) \times \mathrm{H}^{2}(\Omega \backslash \bar{\omega})\right\}, \\
& \boldsymbol{E}_{\mathbf{2}}:=\left\{(\boldsymbol{f}, g) \in\left[\mathbf{H}^{1}(\Omega \backslash \bar{\omega})\right]^{\prime} \times \mathrm{L}^{2}(\Omega \backslash \bar{\omega}) ;(\Phi \boldsymbol{f}, \Phi g) \in \mathbf{H}^{1}(\Omega \backslash \bar{\omega}) \times \mathrm{H}^{2}(\Omega \backslash \bar{\omega})\right\} \times \mathbb{R}^{N} .
\end{aligned}
$$


We adapt the proof of Lemma 3.2 to obtain that $\boldsymbol{F}$ and $\boldsymbol{f}$ are $C^{2}$ in a neighborhood of $\mathbf{0}$. Indeed, since $\boldsymbol{f} \in \mathbf{H}^{3}\left(\mathbb{R}^{N}\right)$, then the function

$$
\boldsymbol{\theta} \in \mathcal{U} \mapsto \boldsymbol{f} \circ(\mathbf{I}+\boldsymbol{\theta}) \in \mathbf{H}^{1}\left(\mathbb{R}^{N}\right)
$$

is $C^{2}$ in a neighborhood of $\mathbf{0}$ (see Lemma 5.3.9 in Ref. 20). Moreover, we check last assumption of Theorem 3.1 (the estimation) like in the proof of Lemma 3.2. We use in particular the local regularity result Appendix A.2. Therefore, the function

$$
\boldsymbol{\theta} \in \mathcal{U} \mapsto\left(\boldsymbol{v}_{\boldsymbol{\theta}}, q_{\theta}\right) \in \mathbf{X}^{1,3}\left(\Omega \backslash \bar{\omega}, \Omega_{\delta} \backslash \bar{\omega}\right) \times \mathrm{X}^{0,2}\left(\Omega \backslash \bar{\omega}, \Omega_{\delta} \backslash \bar{\omega}\right)
$$

is twice differentiable in a neighborhood of $\mathbf{0}$.

Lemma 3.5 (Second order shape differentiability). The solution $(\boldsymbol{u}, p)$ is twice differentiable with respect to the domain.

Proof. Let us set $\boldsymbol{v}_{\boldsymbol{t}}:=\boldsymbol{u}_{\boldsymbol{t}} \circ \phi(t)$ and $q_{t}:=p_{t} \circ \phi(t)$ where $\phi(t)=\mathbf{I}+t \boldsymbol{V}$. Using the Fréchet differentiability Lemma 3.4, we obtain the Gâteaux differentiability in the direction $\boldsymbol{V}$, the functions

$$
t \in[0, T) \mapsto \boldsymbol{v}_{\boldsymbol{t} \mid \Omega_{\delta} \backslash \bar{\omega}} \in \mathbf{H}^{3}\left(\Omega_{\delta} \backslash \bar{\omega}\right) \quad \text { and } \quad t \in[0, T) \mapsto q_{t \mid \Omega_{\delta} \backslash \bar{\omega}} \in \mathrm{H}^{2}\left(\Omega_{\delta} \backslash \bar{\omega}\right)
$$

are two times differentiable at 0 . Using Stein's extension Theorem, there exists

$$
P: \mathbf{H}^{3}\left(\Omega_{\delta} \backslash \bar{\omega}\right) \rightarrow \mathbf{H}^{3}\left(\Omega_{\delta}\right) \text { and } Q: \mathrm{H}^{2}\left(\Omega_{\delta} \backslash \bar{\omega}\right) \rightarrow \mathrm{H}^{2}\left(\Omega_{\delta}\right),
$$

two extension operators linear continuous. Thus, using Lemma 5.3.9 in Ref. 20, the functions

$$
t \in[0, T) \mapsto P\left(\boldsymbol{v}_{\boldsymbol{t}}\right) \circ(\mathbf{I}+t \boldsymbol{V})^{-1}=: \widetilde{\widetilde{\boldsymbol{u}_{\boldsymbol{t}}}} \in \mathbf{H}^{1}\left(\Omega_{\delta}\right)
$$

and

$$
t \in[0, T) \mapsto Q\left(q_{t}\right) \circ(\mathbf{I}+t \boldsymbol{V})^{-1}=: \widetilde{\bar{p}_{t}} \in \mathrm{L}^{2}\left(\Omega_{\delta}\right)
$$

are twice differentiable in a neighborhood of 0 . Moreover, the functions

$$
t \in[0, T) \mapsto \boldsymbol{u}_{\boldsymbol{t} \mid \Omega \backslash \overline{\Omega_{\delta}}}=\boldsymbol{v}_{t|\Omega| \overline{\Omega_{\delta}}} \in \mathbf{H}^{1}\left(\Omega \backslash \overline{\Omega_{\delta}}\right)
$$

and

$$
t \in[0, T) \mapsto p_{t \mid \Omega \backslash \overline{\Omega_{\delta}}}=q_{t \mid \Omega \backslash \overline{\Omega_{\delta}}} \in \mathrm{L}^{2}\left(\Omega \backslash \overline{\Omega_{\delta}}\right)
$$

are twice differentiable in a neighborhood of 0 by composition. Thus, defining

$$
\widetilde{\boldsymbol{u}_{\boldsymbol{t}}}:=\left\{\begin{array}{l}
\widetilde{\boldsymbol{u}_{\boldsymbol{t}}} \text { in } \Omega_{\delta} \\
\boldsymbol{u}_{\boldsymbol{t}} \text { in } \Omega \backslash \overline{\Omega_{\delta}}
\end{array} \text { and } \widetilde{p_{t}}:= \begin{cases}\widetilde{p_{t}} & \text { in } \Omega_{\delta} \\
p_{t} & \text { in } \Omega \backslash \overline{\Omega_{\delta}},\end{cases}\right.
$$

we conclude as in the proof of Lemma 3.3 . 


\section{Proof of the main results}

The identifiability result for Neumann conditions It is directly adapted from Theorem 1.2 in Ref. 4. The main ingredient is the following theorem due to Fabre and Lebeau:

Theorem 4.1 (C. Fabre, G. Lebeau, Ref. 16). Let $\Omega_{0} \subseteq \mathbb{R}^{N}, N \geq 2$, be a bounded domain and $D_{0}$ an open subset of $\Omega_{0}$. If $\boldsymbol{a} \in \mathbf{L}_{\mathrm{loc}}^{\infty}\left(\Omega_{0}\right)$ and $(\boldsymbol{u}, p)$ in $\mathbf{H}_{\text {loc }}^{1}\left(\Omega_{0}\right) \times \mathrm{L}_{\text {loc }}^{2}\left(\Omega_{0}\right)$ is a solution of

$$
\left\{\begin{aligned}
-\Delta \boldsymbol{u}+(\boldsymbol{a} \cdot \nabla) \boldsymbol{u}+\nabla p & =\mathbf{0} \text { in } \Omega_{0}, \\
\operatorname{div} \boldsymbol{u} & =0 \text { in } \Omega_{0},
\end{aligned}\right.
$$

with $\boldsymbol{u}=\mathbf{0}$ in $D_{0}$, then $\boldsymbol{u}=\mathbf{0}$ in $\Omega_{0}$ and $p$ is constant in $\Omega_{0}$.

We deduce from the above theorem the following corollary:

Corollary 4.1 (Unique continuation property). Let $\Omega_{0} \subseteq \mathbb{R}^{N}, N \geq 2$, be a Lipschitz domain. If $(\boldsymbol{u}, p) \in \mathbf{H}^{1}\left(\Omega_{0}\right) \times \mathrm{L}^{2}\left(\Omega_{0}\right)$ is a solution of

$$
\left\{\begin{aligned}
-\nu \Delta \boldsymbol{u}+\nabla p & =\mathbf{0} \text { in } \Omega_{0}, \\
\operatorname{div} \boldsymbol{u} & =0 \text { in } \Omega_{0}, \\
-\nu \partial_{\mathbf{n}} \boldsymbol{u}+p \mathbf{n} & =\mathbf{0} \text { on } O, \\
\boldsymbol{u} & =\mathbf{0} \text { on } O,
\end{aligned}\right.
$$

where $O \subset \partial \Omega_{0}$ is a relatively open non-empty subset, then $\boldsymbol{u}=\mathbf{0}$ and $p=0$ in $\Omega_{0}$.

Proof. (of Corollary 4.1). We enlarge the domain by a ball: we fix a point $y$ in $O$, we set $\rho:=\mathrm{d}(y, \partial O) / 2$ and set $\Omega_{\rho}:=\Omega_{0} \cup \mathcal{B}(y, \rho)$. We define

$$
\widetilde{\boldsymbol{u}}=\left\{\begin{array}{l}
\boldsymbol{u} \text { in } \Omega_{0}, \\
\mathbf{0} \text { in } \Omega_{\rho} \backslash \Omega_{0},
\end{array} \quad \text { and } \quad \widetilde{p}=\left\{\begin{array}{l}
p \text { in } \Omega_{0}, \\
0 \text { in } \Omega_{\rho} \backslash \Omega_{0} .
\end{array}\right.\right.
$$

Since $\boldsymbol{u} \in \mathbf{H}^{1}\left(\Omega_{0}\right)$ and $\boldsymbol{u}=\mathbf{0}$ on $O$, we check that $\widetilde{p} \in \mathrm{L}^{2}\left(\Omega_{\rho}\right)$ and $\widetilde{\boldsymbol{u}} \in \mathbf{H}^{1}\left(\Omega_{\rho}\right)$. By summing on $\Omega_{0}$ and on $\Omega_{\rho}^{e x t}:=\left\{\Omega_{\rho} \cap B(y, \rho)\right\} \backslash O$, we get that for all $\varphi$ in $\boldsymbol{C}_{c}^{\infty}(B(y, \rho))$ :

$$
\int_{\Omega_{\rho}}(\nu \nabla \widetilde{\boldsymbol{u}}: \nabla \boldsymbol{\varphi}-\widetilde{p} \operatorname{div} \boldsymbol{\varphi})=0 .
$$

Then, $-\nu \Delta \widetilde{\boldsymbol{u}}+\nabla \widetilde{p}=\mathbf{0}$ in $\mathcal{D}^{\prime}\left(\Omega_{\rho}\right)$. Proceeding as above, we also get $\operatorname{div} \widetilde{\boldsymbol{u}}=0$ in $\mathcal{D}^{\prime}\left(\Omega_{\rho}\right)$. Moreover, $\widetilde{\boldsymbol{u}}=\mathbf{0}$ in $\Omega_{\rho}^{\mathrm{ext}} \subset \Omega_{\rho}$. Thus, according to the unique continuation Theorem $4.1, \widetilde{\boldsymbol{u}}=\mathbf{0}$ and $\widetilde{p}$ is constant in $\Omega_{\rho}$. Since $\widetilde{p}=0$ in $\Omega_{\rho}^{\mathrm{ext}}, \widetilde{p}=0$ in $\Omega_{\rho}$ then on $\Omega_{0}$ by restriction.

Proof. (of Theorem 2.2). We define $\boldsymbol{u}:=\boldsymbol{u}_{\mathbf{0}}-\boldsymbol{u}_{\mathbf{1}}, p:=p_{0}-p_{1}$ and $\omega:=\omega_{0} \cup \omega_{1}$. Hence, $(\boldsymbol{u}, p)$ satisfies

$$
\left\{\begin{aligned}
-\nu \Delta \boldsymbol{u}+\nabla p & =\mathbf{0} \text { in } \Omega \backslash \bar{\omega} \\
\operatorname{div} \boldsymbol{u} & =0 \text { in } \Omega \backslash \bar{\omega} \\
-\nu \partial_{\mathbf{n}} \boldsymbol{u}+p \mathbf{n} & =\mathbf{0} \text { in } O \\
\boldsymbol{u} & =\mathbf{0} \text { in } O .
\end{aligned}\right.
$$


Therefore, according to Corollary $4.1, \boldsymbol{u}=\mathbf{0}$ and $p=0$ in $\Omega \backslash \bar{\omega}$.

We proceed by contradiction. Assume that $\omega_{0} \backslash \overline{\omega_{1}}$ is non-empty. We know that

$$
-\nu \Delta \boldsymbol{u}_{\mathbf{1}}+\nabla p_{1}=\mathbf{0} \quad \text { in } \omega_{0} \backslash \overline{\omega_{1}} .
$$

Multiplying this equation by $\boldsymbol{u}_{\mathbf{1}}$ and integrating by parts in $\omega_{0} \backslash \overline{\omega_{1}}$ with $\boldsymbol{f} \equiv \mathbf{0}$ in $\Omega_{\delta}$ and $-\nu \partial_{\mathbf{n}} \boldsymbol{u}_{\mathbf{1}}+p_{1} \mathbf{n}=\mathbf{0}$ on $\partial \omega_{1}$, we obtain

$$
\int_{\omega_{0} \backslash \overline{\omega_{1}}} \nu\left|\nabla \boldsymbol{u}_{\mathbf{1}}\right|^{2}+\int_{\left(\partial \omega_{0}\right) \backslash \overline{\omega_{1}}}\left(-\nu \partial_{\mathbf{n}} \boldsymbol{u}_{\mathbf{1}}+p_{1} \mathbf{n}\right) \cdot \boldsymbol{u}_{\mathbf{1}}=0 .
$$

Since $\boldsymbol{u}_{\mathbf{0}}=\boldsymbol{u}_{\mathbf{1}}$ and $p_{0}=p_{1}$ in $\Omega \backslash \bar{\omega}$, the boundary condition satisfied by $\boldsymbol{u}_{\mathbf{0}}$ on $\partial \omega_{0}$ provides

$$
-\nu \partial_{\mathbf{n}} \boldsymbol{u}_{\mathbf{1}}+p_{1} \mathbf{n}=-\nu \partial_{\mathbf{n}} \boldsymbol{u}_{\mathbf{0}}+p_{0} \mathbf{n}=\mathbf{0} \quad \text { on }\left(\partial \omega_{0}\right) \backslash \overline{\omega_{1}} .
$$

Hence, equality (4.2) is simply

$$
\int_{\omega_{0} \backslash \overline{\omega_{1}}} \nu\left|\nabla \boldsymbol{u}_{\mathbf{1}}\right|^{2}=0
$$

Hence, $\boldsymbol{u}_{\mathbf{1}}$ is constant in $\omega_{0} \backslash \overline{\omega_{1}}$. By Theorem 4.1, $\boldsymbol{u}_{\mathbf{1}}$ and $p_{1}$ are then constant in $\Omega \backslash \overline{\omega_{1}}$ and $-\nu \Delta \boldsymbol{u}_{\mathbf{1}}+\nabla p_{1}=\mathbf{0}$ in $\Omega \backslash \overline{\omega_{1}}$. This contradicts $\boldsymbol{f} \neq \mathbf{0}$ and then $\omega_{0} \backslash \overline{\omega_{1}}=\emptyset$.

By symmetry, $\omega_{1} \backslash \overline{\omega_{0}}=\emptyset$. Finally, $\omega_{0}=\omega_{1}$.

First order shape derivatives of the state. We recall the variational formulation of the problem (2.1) in $\Omega \backslash \overline{\omega_{t}}$ : Find $\left(\boldsymbol{u}_{\boldsymbol{t}}, p_{t}\right) \in \mathbf{H}^{1}\left(\Omega \backslash \overline{\omega_{t}}\right) \times \mathrm{L}^{2}\left(\Omega \backslash \overline{\omega_{t}}\right)$ such that

$$
\left\{\begin{array}{rlrl}
\int_{\Omega \backslash \overline{\omega_{t}}}\left\{\nu \nabla \boldsymbol{u}_{\boldsymbol{t}}: \nabla \boldsymbol{\varphi}_{\boldsymbol{t}}-p_{t} \operatorname{div} \boldsymbol{\varphi}_{\boldsymbol{t}}\right\} & =\int_{\Omega \backslash \overline{\omega_{t}}} \boldsymbol{f} \cdot \boldsymbol{\varphi}_{\boldsymbol{t}} \forall \boldsymbol{\varphi}_{\boldsymbol{t}} \in \mathbf{H}^{1}\left(\Omega \backslash \overline{\omega_{t}}\right), \\
\int_{\Omega \backslash \overline{\omega_{t}}} \xi_{t} \operatorname{div} \boldsymbol{u}_{\boldsymbol{t}} & =0 & \forall \xi_{t} \in \mathrm{L}^{2}\left(\Omega \backslash \overline{\omega_{t}}\right), \\
\int_{O} \boldsymbol{u}_{\boldsymbol{t}} & =\int_{O} \boldsymbol{f}_{\boldsymbol{b}} . &
\end{array}\right.
$$

\section{Proof. (of Proposition 2.5).}

First step: first order shape differentiability. Using the Fréchet differentiability Lemma 3.3, we obtain the Gâteaux differentiability in the direction $\boldsymbol{V}$ : there exist $\boldsymbol{u}_{\boldsymbol{t}}$ and $p_{t}$, respective extensions in $\Omega$ of $\boldsymbol{u}_{\boldsymbol{t}}$ and $p_{t}$ such that the functions, defined on $[0, T), t \mapsto \boldsymbol{u}_{\boldsymbol{t}} \in \mathbf{H}^{1}(\Omega)$ and $t \mapsto p_{t} \in \mathrm{L}^{2}(\Omega)$ are differentiable at 0 by composition. We denote by $\boldsymbol{u}^{\prime}$ and $p^{\prime}$ their respective derivative at 0 .

Second step: derivative of the normalization condition. The function

$$
t \mapsto \int_{O} \boldsymbol{u}_{\boldsymbol{t}}=\int_{O} \boldsymbol{f}_{\boldsymbol{b}}
$$

is constant on $[0, T)$. Thus, its derivative is 0 . By differentiation under the sum sign, we get $\int_{O} \boldsymbol{u}^{\prime}=\mathbf{0}$. 
Third step: derivative of the equalities on $\Omega \backslash \overline{\omega_{t}}$. Let $\varphi \in \mathcal{D}(\Omega \backslash \bar{\omega})$. As $\Omega \backslash \bar{\omega}$ is open, we have, for t small enough, $\varphi \in \mathcal{D}\left(\Omega \backslash \overline{\omega_{t}}\right)$. Therefore, using the first line of the variational formulation (4.3), we have for all $t \in[0, T)$

$$
\begin{aligned}
\int_{\Omega \backslash \bar{\omega}} \boldsymbol{f} \cdot \boldsymbol{\varphi} & =\int_{\Omega \backslash \overline{\omega_{t}}} \boldsymbol{f} \cdot \boldsymbol{\varphi}=\int_{\Omega \backslash \overline{\bar{\omega}_{t}}}\left\{\nu \nabla \boldsymbol{u}_{t}: \nabla \boldsymbol{\varphi}-p_{t} \operatorname{div} \boldsymbol{\varphi}\right\} \\
& =\int_{\Omega \backslash \overline{\omega_{t}}}-\left\{\nu \boldsymbol{u}_{\boldsymbol{t}} \cdot \Delta \boldsymbol{\varphi}-p_{t} \operatorname{div} \varphi\right\}=\int_{\Omega \backslash \bar{\omega}}\left\{-\nu \boldsymbol{u}_{\boldsymbol{t}} \cdot \Delta \boldsymbol{\varphi}-p_{t} \operatorname{div} \boldsymbol{\varphi}\right\} .
\end{aligned}
$$

We differentiate with respect to $t$ at $t=0$

$$
\int_{\Omega \backslash \bar{\omega}} \boldsymbol{f} \cdot \boldsymbol{\varphi}=\int_{\Omega \backslash \bar{\omega}}\left\{-\nu \boldsymbol{u}_{\boldsymbol{t}} \cdot \Delta \boldsymbol{\varphi}-p_{t} \operatorname{div} \boldsymbol{\varphi}\right\}
$$

to obtain

$$
0=\int_{\Omega \backslash \bar{\omega}}\left\{-\nu \boldsymbol{u}^{\prime} \cdot \Delta \boldsymbol{\varphi}-p^{\prime} \operatorname{div} \boldsymbol{\varphi}\right\}=\left\langle-\nu \Delta \boldsymbol{u}^{\prime}+\nabla p^{\prime}, \varphi\right\rangle_{\mathcal{D}^{\prime}(\Omega \backslash \bar{\omega}), \mathcal{D}(\Omega \backslash \bar{\omega})} .
$$

As it is true for all $\varphi \in \mathcal{D}(\Omega \backslash \bar{\omega})$, we get

$$
-\nu \Delta \boldsymbol{u}^{\prime}+\nabla p^{\prime}=\mathbf{0} \quad \text { in } \mathcal{D}^{\prime}(\Omega \backslash \bar{\omega}) .
$$

Proceeding with the second line of the variational formulation (4.3) as previously, we check that $\operatorname{div} \boldsymbol{u}^{\prime}=0$ in $\mathcal{D}^{\prime}(\Omega \backslash \bar{\omega})$.

Fourth step: variational characterization of $\boldsymbol{u}^{\prime}$ and $p^{\prime}$. Let $\boldsymbol{\varphi} \in \mathbf{H}^{2}(\Omega \backslash \bar{\omega})$. Using Stein's extension Theorem, $\varphi$ admits an extension in $\mathbf{H}^{2}\left(\mathbb{R}^{N}\right)$ still noted $\varphi$. This extension is in particular in $\mathbf{H}^{2}\left(\Omega \backslash \overline{\omega_{t}}\right)$. Using $\varphi$ as a test function in the first line of the variational formulation (4.3), we have

$$
\int_{\Omega \backslash \overline{\omega_{t}}}\left\{\nu \nabla \boldsymbol{u}_{t}: \nabla \boldsymbol{\varphi}-p_{t} \operatorname{div} \boldsymbol{\varphi}\right\}=\int_{\Omega \backslash \overline{\omega_{t}}} \boldsymbol{f} \cdot \boldsymbol{\varphi} .
$$

To compute

$$
I:=\frac{d}{d t}\left(\int_{\Omega \backslash \overline{\omega_{t}}}\left\{\nu \nabla \boldsymbol{u}_{\boldsymbol{t}}: \nabla \boldsymbol{\varphi}-p_{t} \operatorname{div} \boldsymbol{\varphi}\right\}\right)_{t=0},
$$

we use Hadamard's formula (see Corollary 5.2.5 in Ref. 20). Let us check its assumptions. Set

$$
\eta(t):=\nu\left[\nabla \boldsymbol{v}_{t}(\mathrm{I}+\nabla \boldsymbol{V})^{-1}\right]:[(\nabla \boldsymbol{\varphi}) \circ \phi(t)]-q_{t}[(\operatorname{div} \boldsymbol{\varphi}) \circ \phi(t)]
$$

so that for $t$ in a neighborhood of 0 ,

$$
\nu \nabla \boldsymbol{u}_{\boldsymbol{t}}: \nabla \boldsymbol{\varphi}-p_{t} \operatorname{div} \boldsymbol{\varphi}=\eta(t) \circ \phi(t)^{-1},
$$

(since $p_{t}=q_{t} \circ \phi(t)^{-1}$ and $\left.\nabla \boldsymbol{u}_{\boldsymbol{t}}=\left[\nabla \boldsymbol{v}_{t}(\mathrm{I}+\nabla \boldsymbol{V})^{-1}\right] \circ \phi(t)^{-1}\right)$. Then, we have

- $\nu \nabla \boldsymbol{u}_{t}: \nabla \boldsymbol{\varphi}-p_{t} \operatorname{div} \boldsymbol{\varphi} \in \mathbf{L}^{1}\left(\Omega_{\delta} \backslash \overline{\omega_{t}}\right)$ since $\boldsymbol{\varphi}$ belongs to $\mathbf{H}^{2}\left(\mathbb{R}^{N}\right),\left(\boldsymbol{u}_{t}, p_{t}\right)$ belongs to $\mathbf{H}^{2}\left(\Omega_{\delta} \backslash \overline{\omega_{t}}\right) \times \mathrm{H}^{1}\left(\Omega_{\delta} \backslash \overline{\omega_{t}}\right)$ and $\Omega_{\delta} \backslash \overline{\omega_{t}}$ is bounded,

- $t \mapsto \eta(t) \in \mathrm{L}^{1}\left(\Omega_{\delta} \backslash \bar{\omega}\right)$ is differentiable at 0 by composition since $t \mapsto \phi(t)$ is differentiable at 0 and $\boldsymbol{\theta} \mapsto\left(\boldsymbol{v}_{\boldsymbol{\theta}}, q_{\theta}\right)$ is differentiable at $\mathbf{0}$ using Lemma 3.2, 
- $\nu \nabla \boldsymbol{u}_{0}: \nabla \boldsymbol{\varphi}-p_{0} \operatorname{div} \boldsymbol{\varphi}=\nu \nabla \boldsymbol{u}: \nabla \boldsymbol{\varphi}-p \operatorname{div} \boldsymbol{\varphi} \in \mathbf{W}^{1,1}\left(\Omega_{\delta} \backslash \bar{\omega}\right)$ since $\boldsymbol{\varphi}$ belongs to $\mathbf{H}^{2}\left(\mathbb{R}^{N}\right),(\boldsymbol{u}, p)$ belongs to $\mathbf{H}^{2}\left(\Omega_{\delta} \backslash \bar{\omega}\right) \times \mathrm{H}^{1}\left(\Omega_{\delta} \backslash \bar{\omega}\right)$ and $\Omega_{\delta} \backslash \bar{\omega}$ is bounded.

Thus, using Hadamard's formula (Corollary 5.2.5 in Ref. 20), we decompose the derivative $I$ into $I=J_{1}+J_{2}$ with

$$
\begin{aligned}
& J_{1}:=\int_{\Omega \backslash \overline{\Omega_{\delta}}}\left\{\nu \nabla \boldsymbol{u}^{\prime}: \nabla \boldsymbol{\varphi}-p^{\prime} \operatorname{div} \boldsymbol{\varphi}\right\}, \\
& J_{2}:=\int_{\Omega_{\delta} \backslash \bar{\omega}}\left\{\nu \nabla \boldsymbol{u}^{\prime}: \nabla \boldsymbol{\varphi}-p^{\prime} \operatorname{div} \boldsymbol{\varphi}\right\}+\int_{\partial\left(\Omega_{\delta} \backslash \bar{\omega}\right)}(\nu \nabla \boldsymbol{u}: \nabla \boldsymbol{\varphi}-p \operatorname{div} \boldsymbol{\varphi})(\boldsymbol{V} \cdot \mathbf{n}) .
\end{aligned}
$$

Since $\boldsymbol{V}=0$ on $\partial \Omega_{\delta}$, we have:

$$
J_{2}=\int_{\Omega_{\delta} \backslash \bar{\omega}}\left\{\nu \nabla \boldsymbol{u}^{\prime}: \nabla \boldsymbol{\varphi}-p^{\prime} \operatorname{div} \boldsymbol{\varphi}\right\}+\int_{\partial \omega}(\nu \nabla \boldsymbol{u}: \nabla \boldsymbol{\varphi}-p \operatorname{div} \boldsymbol{\varphi})(\boldsymbol{V} \cdot \mathbf{n}) .
$$

Therefore

$$
I=\int_{\Omega \backslash \bar{\omega}}\left\{\nu \nabla \boldsymbol{u}^{\prime}: \nabla \boldsymbol{\varphi}-p^{\prime} \operatorname{div} \boldsymbol{\varphi}\right\}+\int_{\partial \omega}(\nu \nabla \boldsymbol{u}: \nabla \boldsymbol{\varphi}-p \operatorname{div} \boldsymbol{\varphi})(\boldsymbol{V} \cdot \mathbf{n}) .
$$

By the same technics, we differentiate the right hand side of (4.5):

$$
\frac{d}{d t}\left(\int_{\Omega \backslash \overline{\omega_{t}}} \boldsymbol{f} \cdot \boldsymbol{\varphi}\right)_{t=0}=\int_{\Omega \backslash \bar{\omega}} \operatorname{div}(\boldsymbol{f} \cdot \boldsymbol{\varphi}) V=\int_{\partial \omega}(\boldsymbol{f} \cdot \boldsymbol{\varphi})(\boldsymbol{V} \cdot \mathbf{n}) .
$$

Gathering (4.5), (4.6) and (4.7), we get

$$
\int_{\Omega \backslash \bar{\omega}}\left\{\nu \nabla \boldsymbol{u}^{\prime}: \nabla \boldsymbol{\varphi}-p^{\prime} \operatorname{div} \boldsymbol{\varphi}\right\}+\int_{\partial \omega}(\nu \nabla \boldsymbol{u}: \nabla \boldsymbol{\varphi}-p \operatorname{div} \boldsymbol{\varphi})(\boldsymbol{V} \cdot \mathbf{n})=\int_{\partial \omega}(\boldsymbol{f} \cdot \boldsymbol{\varphi})(\boldsymbol{V} \cdot \mathbf{n}) .
$$

We use the definition of tangential gradient and divergence and the formulae $\left(\partial_{\mathbf{n}} \boldsymbol{u} \otimes \mathbf{n}\right):\left(\partial_{\mathbf{n}} \boldsymbol{\varphi} \otimes \mathbf{n}\right)=\partial_{\mathbf{n}} \boldsymbol{u} \cdot \partial_{\mathbf{n}} \boldsymbol{\varphi}$ and $\nabla \boldsymbol{u}:\left(\partial_{\mathbf{n}} \boldsymbol{\varphi} \otimes \mathbf{n}\right)=\partial_{\mathbf{n}} \boldsymbol{u} \cdot \partial_{\mathbf{n}} \boldsymbol{\varphi}=\nabla \boldsymbol{\varphi}:\left(\partial_{\mathbf{n}} \boldsymbol{u} \otimes \mathbf{n}\right)$ to get

$$
\nabla \boldsymbol{u}: \nabla \boldsymbol{\varphi}=\nabla_{\Gamma} \boldsymbol{u}: \nabla_{\Gamma} \boldsymbol{\varphi}+\partial_{\mathbf{n}} \boldsymbol{u} \cdot \partial_{\mathbf{n}} \boldsymbol{\varphi} .
$$

Plugging this relation in (4.8), we obtain:

$$
\begin{aligned}
\int_{\Omega \backslash \omega}\{\nu & \left.\nabla \boldsymbol{u}^{\prime}: \nabla \boldsymbol{\varphi}-p^{\prime} \operatorname{div} \boldsymbol{\varphi}\right\}+\int_{\partial \omega}\left(\nu \nabla_{\Gamma} \boldsymbol{u}: \nabla_{\Gamma} \boldsymbol{\varphi}-p \operatorname{div}_{\Gamma} \boldsymbol{\varphi}\right)(\boldsymbol{V} \cdot \mathbf{n}) \\
& =\int_{\partial \omega}\left\{(\boldsymbol{f} \cdot \boldsymbol{\varphi})(\boldsymbol{V} \cdot \mathbf{n})-\nu \partial_{\mathbf{n}} \boldsymbol{u} \cdot \partial_{\mathbf{n}} \boldsymbol{\varphi}(\boldsymbol{V} \cdot \mathbf{n})+\partial_{\mathbf{n}} \boldsymbol{\varphi} \cdot(p \mathbf{n})(\boldsymbol{V} \cdot \mathbf{n})\right\} .
\end{aligned}
$$

We use the boundary condition $-\nu \partial_{\mathbf{n}} \boldsymbol{u}+p \mathbf{n}=\mathbf{0}$ on $\partial \omega$ to simplify this relation in:

$$
\int_{\Omega \backslash \bar{\omega}}\left\{\nu \nabla \boldsymbol{u}^{\prime}: \nabla \boldsymbol{\varphi}-p^{\prime} \operatorname{div} \boldsymbol{\varphi}\right\}+\int_{\partial \omega}\left(\nu \nabla_{\Gamma} \boldsymbol{u}: \nabla_{\Gamma} \boldsymbol{\varphi}-p \operatorname{div}_{\Gamma} \boldsymbol{\varphi}\right)(\boldsymbol{V} \cdot \mathbf{n})=\int_{\partial \omega}(\boldsymbol{f} \cdot \boldsymbol{\varphi})(\boldsymbol{V} \cdot \mathbf{n}) .
$$

Fifth step: derivative of the boundary values condition. We differentiate with respect to $t$ the relation $-\nu \partial_{\mathbf{n}} \boldsymbol{u}_{\boldsymbol{t}}+p_{t} \mathbf{n}=\mathbf{0}$ on $\partial \Omega$. At $t=0$, we obtain on $\partial \Omega$ that $-\nu \partial_{\mathbf{n}} \boldsymbol{u}^{\prime}+p^{\prime} \mathbf{n}=\mathbf{0}$. 
Let us set $\boldsymbol{v}_{\boldsymbol{t}}:=\boldsymbol{u}_{\boldsymbol{t}} \circ \phi(t)$. By differentiation with respect to $t$ (see Corollary 5.2.5 in Ref. 20), we obtain $\boldsymbol{u}^{\prime}=\boldsymbol{v}^{\prime}-\nabla \boldsymbol{u} \boldsymbol{V}$ on $\Omega_{\delta} \backslash \bar{\omega}$, where $\boldsymbol{v}^{\prime}$ is the material derivative of $\boldsymbol{v}_{\boldsymbol{t}}$ at $t=0$. Thus, $\boldsymbol{u}^{\prime}$ belongs to $\mathbf{H}^{2}\left(\Omega_{\delta} \backslash \bar{\omega}\right)$ as a sum of two terms in this space. Indeed, the derivative $\boldsymbol{v}^{\prime}$ is the limit of the differential quotient $\left(\boldsymbol{v}_{\boldsymbol{t}}-\boldsymbol{v}\right) / t$ that belongs to $\mathbf{H}^{2}\left(\Omega_{\delta} \backslash \bar{\omega}\right)$ since $\boldsymbol{v}_{\boldsymbol{t}} \in \mathbf{H}^{2}\left(\Omega_{\delta} \backslash \bar{\omega}\right)$ for all $t \in[0, T)$. The second term $\nabla \boldsymbol{u} \boldsymbol{V}$ is also in $\mathbf{H}^{2}\left(\Omega_{\delta} \backslash \bar{\omega}\right)$ by the local regularity result Appendix A.2. The crucial point here is the $C^{2,1}$ regularity of $\partial \omega$. Therefore, $\Delta \boldsymbol{u}^{\prime} \in \mathbf{L}^{2}\left(\Omega_{\delta} \backslash \bar{\omega}\right)$ and, using (4.4), $\nabla p^{\prime} \in \mathbf{L}^{2}\left(\Omega_{\delta} \backslash \bar{\omega}\right)$.

Now, consider $\varphi \in \mathbf{H}^{2}\left(\Omega_{\delta} \backslash \bar{\omega}\right)$ with compact support in $\Omega_{\delta}$ and compute

$$
\begin{aligned}
\int_{\Omega \backslash \bar{\omega}}\left\{\nu \nabla \boldsymbol{u}^{\prime}: \nabla \boldsymbol{\varphi}-p^{\prime} \operatorname{div} \varphi\right\} & =\int_{\Omega_{\delta} \backslash \bar{\omega}}\left\{\nu \nabla \boldsymbol{u}^{\prime}: \nabla \boldsymbol{\varphi}-p^{\prime} \operatorname{div} \boldsymbol{\varphi}\right\} \\
& =\int_{\Omega_{\delta} \backslash \bar{\omega}}\left(-\nu \Delta \boldsymbol{u}^{\prime}+\nabla p^{\prime}\right) \cdot \boldsymbol{\varphi}+\int_{\partial\left(\Omega_{\delta} \backslash \bar{\omega}\right)}\left(\nu \partial_{\mathbf{n}} \boldsymbol{u}^{\prime}-p^{\prime} \mathbf{n}\right) \cdot \boldsymbol{\varphi} \\
& =\int_{\partial\left(\Omega_{\delta} \backslash \bar{\omega}\right)}\left(\nu \partial_{\mathbf{n}} \boldsymbol{u}^{\prime}-p^{\prime} \mathbf{n}\right) \cdot \boldsymbol{\varphi} \quad \text { by }(4.4) .
\end{aligned}
$$

Since $\boldsymbol{\varphi}=\mathbf{0}$ on $\partial \Omega_{\delta}$, the boundary term on $\partial \Omega_{\delta}$ cancels. Inserting (4.9), we obtain

$$
\begin{aligned}
\int_{\partial \omega}\left(\nu \partial_{\mathbf{n}} \boldsymbol{u}^{\prime}-p^{\prime} \mathbf{n}\right) \cdot \boldsymbol{\varphi}+\int_{\partial \omega}\left(\nu \nabla_{\Gamma} \boldsymbol{u}: \nabla_{\Gamma} \boldsymbol{\varphi}-p \operatorname{div}_{\Gamma} \boldsymbol{\varphi}\right)(\boldsymbol{V} \cdot \mathbf{n}) & \\
& =\int_{\partial \omega} \boldsymbol{f} \cdot \boldsymbol{\varphi}(\boldsymbol{V} \cdot \mathbf{n}) .
\end{aligned}
$$

We integrate by parts on the boundary $\partial \omega$ (which has no boundary)(see Proposition 5.4.9 in Ref. 20) to get

$$
\begin{aligned}
& \int_{\partial \omega} p \operatorname{div}_{\Gamma} \boldsymbol{\varphi}(\boldsymbol{V} \cdot \mathbf{n})=\int_{\partial \omega}\left\{\mathrm{H} p(\boldsymbol{V} \cdot \mathbf{n}) \boldsymbol{\varphi} \cdot \mathbf{n}-\boldsymbol{\varphi} \cdot \nabla_{\Gamma}(p(\boldsymbol{V} \cdot \mathbf{n}))\right\} \\
& \int_{\partial \omega}-\nu \nabla_{\Gamma} \boldsymbol{u}: \nabla_{\Gamma} \boldsymbol{\varphi}(\boldsymbol{V} \cdot \mathbf{n})= \\
& \quad \int_{\partial \omega}\left\{-\boldsymbol{\varphi} \cdot \operatorname{div}_{\Gamma}\left(-\nu \nabla_{\Gamma} \boldsymbol{u}(\boldsymbol{V} \cdot \mathbf{n})\right)+\mathrm{H} \boldsymbol{\varphi} \cdot\left(-\nu \nabla_{\Gamma} \boldsymbol{u}(\boldsymbol{V} \cdot \mathbf{n}) \mathbf{n}\right)\right\},
\end{aligned}
$$

where $\mathrm{H}$ is the mean curvature of $\partial \omega$. Inserting these results in (4.10), we obtain

$$
\begin{aligned}
-\nu \partial_{\mathbf{n}} \boldsymbol{u}^{\prime}+p^{\prime} \mathbf{n}=-\boldsymbol{f}(\boldsymbol{V} \cdot \mathbf{n})- & \operatorname{div}_{\Gamma}\left(\nu \nabla_{\Gamma} \boldsymbol{u}(\boldsymbol{V} \cdot \mathbf{n})\right)+\nu \mathrm{H} \nabla_{\Gamma} \boldsymbol{u}(\boldsymbol{V} \cdot \mathbf{n}) \mathbf{n} \\
& -\mathrm{H} p(\boldsymbol{V} \cdot \mathbf{n}) \mathbf{n}+(\boldsymbol{V} \cdot \mathbf{n}) \nabla_{\Gamma} p+p \nabla_{\Gamma}(\boldsymbol{V} \cdot \mathbf{n}) .
\end{aligned}
$$

We used the fact that $\left.\nabla_{\Gamma}(p(\boldsymbol{V} \cdot \mathbf{n}))=(\boldsymbol{V} \cdot \mathbf{n}) \nabla_{\Gamma} p+p \nabla_{\Gamma}(\boldsymbol{V} \cdot \mathbf{n})\right)$ and the density in $\mathbf{L}^{2}(\partial \omega)$ of the traces on $\partial \omega$ of functions $\mathbf{H}^{2}\left(\Omega_{\delta} \backslash \bar{\omega}\right)$ with compact support in $\Omega_{\delta}$.

Let us simplify this expression. According to the definition of the tangential gradient (see (1.1)), $\nabla_{\Gamma} \boldsymbol{u} \nabla_{\Gamma}(\boldsymbol{V} \cdot \mathbf{n})=\nabla \boldsymbol{u} \nabla_{\Gamma}(\boldsymbol{V} \cdot \mathbf{n})$. We expand the tangential divergence

$$
\operatorname{div}_{\Gamma}\left(\nu \nabla_{\Gamma} \boldsymbol{u}(\boldsymbol{V} \cdot \mathbf{n})\right)=\nu \operatorname{div}_{\Gamma}\left(\nabla_{\Gamma} \boldsymbol{u}\right)(\boldsymbol{V} \cdot \mathbf{n})+\nu \nabla_{\Gamma} \boldsymbol{u} \nabla_{\Gamma}(\boldsymbol{V} \cdot \mathbf{n})
$$


and obtain the decomposition

$$
\operatorname{div}_{\Gamma}\left(\nu \nabla_{\Gamma} \boldsymbol{u}(\boldsymbol{V} \cdot \mathbf{n})\right)=\nu \Delta_{\Gamma} \boldsymbol{u}(\boldsymbol{V} \cdot \mathbf{n})+\nu \nabla \boldsymbol{u} \nabla_{\Gamma}(\boldsymbol{V} \cdot \mathbf{n}) .
$$

Using Stokes equations $-\nu \Delta \boldsymbol{u}+\nabla p=\boldsymbol{f}$ in $\Omega \backslash \bar{\omega}$ and the decomposition of the Laplace-Beltrami operator (see Proposition 5.4.12 in Ref. 20), we compute

$$
\boldsymbol{f}=-\nu\left(\Delta_{\Gamma} \boldsymbol{u}+\mathrm{H} \partial_{\mathbf{n}} \boldsymbol{u}+\partial_{\mathbf{n n}}^{2} \boldsymbol{u}\right)+\nabla p \text { on } \partial \omega .
$$

Inserting (4.12) and (4.13) in (4.11), we obtain

$$
\begin{aligned}
-\nu \partial_{\mathbf{n}} \boldsymbol{u}^{\prime}+p^{\prime} \mathbf{n}=\nu & \partial_{\mathbf{n n}}^{2} \boldsymbol{u}(\boldsymbol{V} \cdot \mathbf{n})-\nabla p(\boldsymbol{V} \cdot \mathbf{n})-\nu \nabla \boldsymbol{u} \nabla_{\Gamma}(\boldsymbol{V} \cdot \mathbf{n}) \\
& +\nu \mathrm{H} \nabla_{\Gamma} \boldsymbol{u}(\boldsymbol{V} \cdot \mathbf{n}) \mathbf{n}+(\boldsymbol{V} \cdot \mathbf{n}) \nabla_{\Gamma} p+p \nabla_{\Gamma}(\boldsymbol{V} \cdot \mathbf{n}) \quad \text { on } \partial \omega,
\end{aligned}
$$

since $(\boldsymbol{u}, p)$ satisfies the boundary conditions $-\nu \partial_{\mathbf{n}} \boldsymbol{u}+p \mathbf{n}=\mathbf{0}$ on $\partial \omega$. We conclude the proof by noting that $\nabla_{\Gamma} \boldsymbol{u} \mathbf{n}=\mathbf{0}$.

First order shape derivatives of the functional. Define, for all $t \in[0, T)$,

$$
J\left(\omega_{t}\right):=j(t):=\int_{O}\left(\boldsymbol{u}_{t}-\boldsymbol{f}_{\boldsymbol{b}}\right)^{2} .
$$

\section{Proof. (of Proposition 2.6).}

First step: derivative of $j$ and adjoint problem. By Proposition 2.5, $(\boldsymbol{u}, p)$ is differentiable with respect to the shape. We denote their respective derivative by $\boldsymbol{u}^{\prime}$ and $p^{\prime}$. Differentiating $j$ with respect to $t$ at 0 , we obtain

$$
j^{\prime}(0)=\int_{O} 2 \boldsymbol{u}^{\prime}\left(\boldsymbol{u}-\boldsymbol{f}_{\boldsymbol{b}}\right) .
$$

Then, we consider the adjoint problem (2.3). According to Appendix A.1, it admits a unique solution $(\boldsymbol{w}, q) \in \mathbf{H}^{1}(\Omega \backslash \bar{\omega}) \times \mathrm{L}^{2}(\Omega \backslash \bar{\omega})$ with $\int_{O} \boldsymbol{w}=\mathbf{0}$. Indeed, the compatibility condition for the adjoint is true as consequence of our choice of compatibility condition for the state.

Second step: writing of $j^{\prime}(0)$ as an integral on $\partial \omega$. We proceed by successive integrations by parts. We multiply the first equation of the adjoint problem (2.3) by $\boldsymbol{u}^{\prime}$ to get:

$$
\int_{\Omega \backslash \bar{\omega}} \nu \nabla \boldsymbol{w}: \nabla \boldsymbol{u}^{\prime}=-\left\langle-\nu \partial_{\mathbf{n}} \boldsymbol{w}+q \mathbf{n}, \boldsymbol{u}^{\prime}\right\rangle_{\partial(\Omega \backslash \bar{\omega})},
$$

since $\operatorname{div} \boldsymbol{u}^{\prime}=0$ in $\Omega \backslash \bar{\omega}$ (see Proposition 2.5). Then, we multiply the first equation of the problem (2.2) by $\boldsymbol{w}$ to obtain

$$
\int_{\Omega \backslash \bar{\omega}} \nu \nabla \boldsymbol{u}^{\prime}: \nabla \boldsymbol{w}=-\int_{\partial(\Omega \backslash \bar{\omega})}\left(-\nu \partial_{\mathbf{n}} \boldsymbol{u}^{\prime}+p^{\prime} \mathbf{n}\right) \cdot \boldsymbol{w},
$$


since $\operatorname{div} \boldsymbol{w}=0$ in $\Omega \backslash \bar{\omega}$. Therefore, gathering (4.14) and (4.15) and using the boundary conditions of $\left(\boldsymbol{u}^{\prime}, p^{\prime}\right)$ and $(\boldsymbol{w}, q)$ (see problems (2.2) and (2.3)), we obtain

$$
\begin{aligned}
\int_{O} 2\left(\boldsymbol{u}-\boldsymbol{f}_{\boldsymbol{b}}\right) \boldsymbol{u}^{\prime} & =\int_{\partial \omega}\left[\left(\nu \partial_{\mathbf{n n}}^{2} \boldsymbol{u}-\partial_{\mathbf{n}} p \mathbf{n}\right)(\boldsymbol{V} \cdot \mathbf{n})+p \nabla_{\Gamma}(\boldsymbol{V} \cdot \mathbf{n})-\nu \nabla \boldsymbol{u} \nabla_{\Gamma}(\boldsymbol{V} \cdot \mathbf{n})\right] \cdot \boldsymbol{w} \\
& =\int_{\partial \omega}\left\{\left(\nu \partial_{\mathbf{n n}}^{2} \boldsymbol{u}-\partial_{\mathbf{n}} p \mathbf{n}\right) \cdot \boldsymbol{w}(\boldsymbol{V} \cdot \mathbf{n})+\left[{ }^{t}(p \mathrm{I}-\nu \nabla \boldsymbol{u}) \boldsymbol{w}\right] \cdot \nabla_{\Gamma}(\boldsymbol{V} \cdot \mathbf{n})\right\} .
\end{aligned}
$$

Finally, we integrate by parts on the boundary $\partial \omega$ to obtain

$$
\int_{O} 2\left(\boldsymbol{u}-\boldsymbol{f}_{\boldsymbol{b}}\right) \boldsymbol{u}^{\prime}=\int_{\partial \omega}\left[\left(\nu \partial_{\mathbf{n n}}^{2} \boldsymbol{u}-\partial_{\mathbf{n}} p \mathbf{n}\right) \cdot \boldsymbol{w}-\operatorname{div}_{\Gamma}\left(\left(p \mathrm{I}-\nu^{t} \nabla \boldsymbol{u}\right) \boldsymbol{w}\right)\right](\boldsymbol{V} \cdot \mathbf{n}) .
$$

Characterization of the shape Hessian at a critical point. We consider $\omega^{*}$ a critical shape of the functional $J$. We assume that $\omega^{*}$ has a $C^{2,1}$ boundary. Thanks to the local regularity result Appendix A.2,

$$
(\boldsymbol{u}, p) \in \mathbf{H}^{3}\left(\Omega_{\delta} \backslash \overline{\omega^{*}}\right) \times \mathrm{H}^{2}\left(\Omega_{\delta} \backslash \overline{\omega^{*}}\right) \quad \text { and } \quad\left(\boldsymbol{u}^{\prime}, p^{\prime}\right) \in \mathbf{H}^{2}\left(\Omega_{\delta} \backslash \overline{\omega^{*}}\right) \times \mathrm{H}^{1}\left(\Omega_{\delta} \backslash \overline{\omega^{*}}\right) .
$$

\section{Proof. (of Proposition 2.7).}

First step : second order shape differentiability. By Lemma 3.5, $(\boldsymbol{u}, p)$ is twice differentiable with respect to the domain. We denote by $\left(\boldsymbol{u}^{\prime \prime}, p^{\prime \prime}\right)$ the second order shape derivatives.

Second step: second derivative of $j$ and derivative of the adjoint problem. Consider $\boldsymbol{V} \in \boldsymbol{U}$. We differentiate the function $j$ twice with respect to $t$. At $t=0$, it holds

$$
j^{\prime \prime}(0)=\mathrm{D}^{2} J(\omega) \cdot \boldsymbol{V} \cdot \boldsymbol{V}=\int_{O}\left\{2\left(\boldsymbol{u}^{\prime}\right)^{2}+2 \boldsymbol{u}^{\prime \prime}\left(\boldsymbol{u}-\boldsymbol{f}_{\boldsymbol{b}}\right)\right\} .
$$

Since $\omega^{*}$ solves the inverse problem, $\boldsymbol{u}=\boldsymbol{f}_{\boldsymbol{b}}$ on $O$. Therefore

$$
\mathrm{D}^{2} J\left(\omega^{*}\right) \cdot \boldsymbol{V} \cdot \boldsymbol{V}=\int_{O} 2\left(\boldsymbol{u}^{\prime}\right)^{2}
$$

We introduce $(\boldsymbol{w}, q) \in \mathbf{H}^{1}(\Omega \backslash \bar{\omega}) \times \mathrm{L}^{2}(\Omega \backslash \bar{\omega})$ with $\int_{O} \boldsymbol{w}=\mathbf{0}$ the solution of the adjoint system (2.3). As we characterized $\boldsymbol{u}^{\prime}$ and $p^{\prime}$ (see Proposition 2.5), we characterize $\boldsymbol{w}^{\prime}$ and $q^{\prime}$, the shape derivatives of $\boldsymbol{w}$ and $q$ to get

$-\nu \partial_{\mathbf{n}} \boldsymbol{w}^{\prime}+q^{\prime} \mathbf{n}=\left(\nu \partial_{\mathbf{n n}}^{2} \boldsymbol{w}-\partial_{\mathbf{n}} q \mathbf{n}\right)(\boldsymbol{V} \cdot \mathbf{n})+q \nabla_{\Gamma}(\boldsymbol{V} \cdot \mathbf{n})-\nu \nabla \boldsymbol{w} \nabla_{\Gamma}(\boldsymbol{V} \cdot \mathbf{n}) \quad$ on $\partial \omega$.

In particular, for $\omega=\omega^{*}, \boldsymbol{u}=\boldsymbol{f}_{\boldsymbol{b}}$ on $O$. Uniqueness of the solution of Stokes problem then enforces that $(\boldsymbol{w}, q)=(\boldsymbol{c}, 0)$ where $\boldsymbol{c}$ is constant. Using the fact that $\boldsymbol{w}=\boldsymbol{c}$ and $q=0$, we obtain system (2.4).

Third step: writing of $j^{\prime \prime}(0)$ as an integral on $\partial \omega$. We multiply the first equation of problem (2.4) by $\boldsymbol{u}^{\prime}$ to get

$$
\int_{\Omega \backslash \omega^{*}} \nu \nabla \boldsymbol{w}^{\prime}: \nabla \boldsymbol{u}^{\prime}=-\left\langle-\nu \partial_{\mathbf{n}} \boldsymbol{w}^{\prime}+q^{\prime} \mathbf{n}, \boldsymbol{u}^{\prime}\right\rangle_{\partial \Omega} .
$$


We multiply the first equation of problem (2.2) by $\boldsymbol{w}^{\prime}$ to get

$$
\int_{\Omega \backslash \omega^{*}} \nu \nabla \boldsymbol{u}^{\prime}: \nabla \boldsymbol{w}^{\prime}=-\left\langle-\nu \partial_{\mathbf{n}} \boldsymbol{u}^{\prime}+p^{\prime} \mathbf{n}, \boldsymbol{w}^{\prime}\right\rangle_{\partial \omega^{*}} .
$$

Therefore, gathering (4.16) and (4.17), we obtain

$$
\int_{O} 2\left(\boldsymbol{u}^{\prime}\right)^{2}=\int_{\partial \omega^{*}}\left[\left(\nu \partial_{\mathbf{n n}}^{2} \boldsymbol{u}-\partial_{\mathbf{n}} p \mathbf{n}\right)(\boldsymbol{V} \cdot \mathbf{n})+p \nabla_{\Gamma}(\boldsymbol{V} \cdot \mathbf{n})-\nu \nabla \boldsymbol{u} \nabla_{\Gamma}(\boldsymbol{V} \cdot \mathbf{n})\right] \cdot \boldsymbol{w}_{\sqcup}^{\prime}
$$

Justifying the ill-posedness of the problem. The compactness of the Riesz operator corresponding to the shape Hessian at possible solutions of the inverse problem is here proved using a local regularity argument. We do not use the hydrodynamical potential layers as it was done in Ref. 2 for the Laplacian case. This should provide an alternative proof.

To prove Proposition 2.8, we first decompose the shape Hessian at a critical point: for $\boldsymbol{V} \in \boldsymbol{U}$,

$$
\mathrm{D}^{2} J\left(\omega^{*}\right) \cdot \boldsymbol{V} \cdot \boldsymbol{V}=\left\langle T_{\boldsymbol{u}, p}(\boldsymbol{V}), M_{2} \circ M_{1}(\boldsymbol{V})\right\rangle_{\partial \omega^{*}} .
$$

Here, $T_{\boldsymbol{u}, p}: \mathbf{H}^{1 / 2}\left(\partial \omega^{*}\right) \rightarrow \mathbf{H}^{-1 / 2}\left(\partial \omega^{*}\right)$ is defined by

$$
T_{\boldsymbol{u}, p}(\boldsymbol{V})=\left(\nu \partial_{\mathbf{n n}}^{2} \boldsymbol{u}-\partial_{\mathbf{n}} p \mathbf{n}\right)(\boldsymbol{V} \cdot \mathbf{n})+p \nabla_{\Gamma}(\boldsymbol{V} \cdot \mathbf{n})-\nu \nabla \boldsymbol{u} \nabla_{\Gamma}(\boldsymbol{V} \cdot \mathbf{n}),
$$

and where $M_{1}: \mathbf{H}^{1 / 2}\left(\partial \omega^{*}\right) \rightarrow \mathbf{H}^{1 / 2}(\partial \Omega)$ is defined by $M_{1}(\boldsymbol{V})=\boldsymbol{u}^{\prime}$, the solution of (2.2). Finally, $M_{2}: \mathbf{H}^{1 / 2}(\partial \Omega) \rightarrow \mathbf{H}^{1 / 2}\left(\partial \omega^{*}\right)$ satisfies $M_{2}(\boldsymbol{\varphi})=\boldsymbol{\psi}$, where $\boldsymbol{\psi}$ is the trace on $\partial \omega^{*}$ of the solution $\boldsymbol{v}$ of the following Stokes problem

$$
\left\{\begin{aligned}
-\nu \Delta \boldsymbol{v}+\nabla \chi & =\mathbf{0} & & \text { in } \Omega \backslash \overline{\omega^{*}} \\
\operatorname{div} \boldsymbol{v} & =0 & & \text { in } \Omega \backslash \overline{\omega^{*}} \\
-\nu \partial_{\mathbf{n}} \boldsymbol{v}+\chi \mathbf{n} & =2 \varphi \mathbb{1}_{O} & & \text { on } \partial \Omega \\
-\nu \partial_{\mathbf{n}} \boldsymbol{v}+\chi \mathbf{n} & =\mathbf{0} & & \text { on } \partial \omega^{*}
\end{aligned}\right.
$$

We study each operator: Lemma 4.1 states that $T_{u, p}$ and $M_{1}$ are linear continuous and Lemma 4.2 claims that $M_{2}$ is compact. Hence, compactness is obtained by composition of linear continuous operator with a compact one.

Lemma 4.1. The operators $T_{\boldsymbol{u}, p}$ and $M_{1}$ are linear continuous.

Proof. Since $\boldsymbol{u}$ and $p$ don't depend on $\boldsymbol{V}$ and using the continuity of the tangential gradient operator $\nabla_{\Gamma}$, the operator $T_{\boldsymbol{u}, p}$ is linear continuous as multiplier by a smooth function (see Ref. 21).

Let $\boldsymbol{V} \in \mathbf{H}^{1 / 2}\left(\partial \omega^{*}\right)$. We define $M_{1}(\boldsymbol{V})=: \boldsymbol{u}^{\prime}$ and

$$
\boldsymbol{g}_{\boldsymbol{b}}(\boldsymbol{V}):=\left(\nu \partial_{\mathbf{n n}}^{2} \boldsymbol{u}-\partial_{\mathbf{n}} p \mathbf{n}\right)(\boldsymbol{V} \cdot \mathbf{n})+p \nabla_{\Gamma}(\boldsymbol{V} \cdot \mathbf{n})-\nu \nabla \boldsymbol{u} \nabla_{\Gamma}(\boldsymbol{V} \cdot \mathbf{n}) .
$$

Since $\nabla_{\Gamma}$ is linear continuous from $\mathbf{H}^{1 / 2}\left(\partial \omega^{*}\right)$ into $\mathbf{H}^{-1 / 2}\left(\partial \omega^{*}\right)$,

$$
\varphi_{1}: \boldsymbol{V} \in \mathbf{H}^{1 / 2}\left(\partial \omega^{*}\right) \mapsto \boldsymbol{g}_{\boldsymbol{b}}(\boldsymbol{V}) \in \mathbf{H}^{-1 / 2}\left(\partial \omega^{*}\right)
$$


is linear continuous. Moreover the following operator is linear continuous:

$$
\varphi_{2}: \boldsymbol{B} \in \mathbf{H}^{-1 / 2}\left(\partial \omega^{*}\right) \mapsto \boldsymbol{\psi} \in \mathbf{H}^{1 / 2}(\partial \Omega),
$$

where $\boldsymbol{\psi}$ is the trace on $\partial \Omega$ of the solution $\boldsymbol{v}$ of the problem

$$
\left\{\begin{aligned}
-\nu \Delta \boldsymbol{v}+\nabla \pi=\mathbf{0} & \text { in } \Omega \backslash \overline{\omega^{*}} \\
\operatorname{div} \boldsymbol{v}=0 & \text { in } \Omega \backslash \overline{\omega^{*}} \\
-\nu \partial_{\mathbf{n}} \boldsymbol{v}+\pi \mathbf{n}=\mathbf{0} & \text { on } \partial \Omega \\
-\nu \partial_{\mathbf{n}} \boldsymbol{v}+\pi \mathbf{n}=\boldsymbol{B} & \text { on } \partial \omega^{*}
\end{aligned}\right.
$$

with $\int_{O} \boldsymbol{v}=\mathbf{0}$. Finally, by composition, $M_{1}=\varphi_{2} \circ \varphi_{1}$ is linear continuous from $\mathbf{H}^{1 / 2}\left(\partial \omega^{*}\right)$ into $\mathbf{H}^{1 / 2}(\partial \Omega)$.

Lemma 4.2. The operator $M_{2}: \mathbf{H}^{1 / 2}(\partial \Omega) \rightarrow \mathbf{H}^{1 / 2}\left(\partial \omega^{*}\right)$ is compact.

Proof. According to the local regularity result Appendix A.2, the operator

$$
\boldsymbol{\varphi} \in \mathbf{H}^{1 / 2}(\partial \Omega) \mapsto \boldsymbol{v} \in \mathbf{H}^{2}\left(\Omega_{\delta} \backslash \overline{\omega^{*}}\right)
$$

is linear continuous. Moreover, the domain $\Omega_{\delta} \backslash \overline{\omega^{*}}$ is bounded and the embedding of $\mathbf{H}^{2}\left(\Omega_{\delta} \backslash \overline{\omega^{*}}\right)$ in $\mathbf{H}^{1}\left(\Omega_{\delta} \backslash \overline{\omega^{*}}\right)$ is then compact. Thus, since the trace operator of $\partial \omega^{*}$ from $\mathbf{H}^{1}\left(\Omega_{\delta} \backslash \overline{\omega^{*}}\right)$ to $\mathbf{H}^{1 / 2}\left(\partial \omega^{*}\right)$ is linear continuous, we get, by composition, that $M_{2}$ is compact.

\section{Appendix A. Some results on the Stokes problem with Neumann conditions}

In order to be complete, we choose to recall classical results with short proofs about the Stokes problem with Neumann conditions: a theorem of existence and uniqueness of the solution and a local regularity result. We refer to Ref. 9 and Ref. 18 for a detailed study, in particular for the regularity of the solutions.

In all this appendix, we note $C$ a generic positive constant only depending on the geometry of the domain and which may change from line to line.

For beginning, let us introduce two notations: for $\Omega$ an open set of $\mathbb{R}^{N}$, we define

$$
\mathbf{L}_{0}^{2}(\Omega):=\left\{\boldsymbol{v} \in \mathbf{L}^{2}(\Omega) ; \int_{\Omega} \boldsymbol{v}=\mathbf{0}\right\}
$$

and

$$
\boldsymbol{V}^{1}(\Omega):=\left\{\boldsymbol{u} \in \mathbf{H}^{1}(\Omega) ; \operatorname{div} \boldsymbol{u}=0\right\} .
$$

Theorem Appendix A.1 (Existence and uniqueness of the solution). Let $\Omega$ a bounded connected Lipschitz open set of $\mathbb{R}^{N}\left(N \in \mathbb{N}^{*}\right)$ and $\nu>0$. Let $\boldsymbol{f}=\left(f_{1}, \ldots, f_{N}\right) \in\left[\mathbf{H}^{1}(\Omega)\right]^{\prime}, g \in \mathrm{L}^{2}(\Omega)$ and $\boldsymbol{f}_{\boldsymbol{b}}=\left(f_{b_{1}}, \ldots, f_{b_{N}}\right) \in \mathbf{H}^{-1 / 2}(\partial \Omega)$ such 
that $\left\langle f_{i}, 1\right\rangle_{\Omega}=\left\langle f_{b_{i}}, 1\right\rangle_{\partial \Omega}$ for all $i=1, \ldots, N$. Then, the problem: find $(\boldsymbol{u}, p)$ in $\left[\mathbf{H}^{1}(\Omega) \cap \mathbf{L}_{0}^{2}(\Omega)\right] \times \mathrm{L}^{2}(\Omega)$ such that

$$
\left\{\begin{aligned}
\int_{\Omega} \nu \nabla \boldsymbol{u}: \nabla \boldsymbol{v}-\int_{\Omega} p \operatorname{div} \boldsymbol{v} & =\langle\boldsymbol{f}, \boldsymbol{v}\rangle_{\Omega}-\left\langle\boldsymbol{f}_{\boldsymbol{b}}, \boldsymbol{v}\right\rangle_{\partial \Omega} & & \forall \boldsymbol{v} \in \mathbf{H}^{1}(\Omega), \\
\int_{\Omega} \xi \operatorname{div} \boldsymbol{u} & =\int_{\Omega} \xi g & & \forall \xi \in \mathrm{L}^{2}(\Omega),
\end{aligned}\right.
$$

admits a unique solution and the following estimate holds:

$$
\|\boldsymbol{u}\|_{\mathbf{H}^{1}(\Omega)}+\|p\|_{\mathrm{L}^{2}(\Omega)} \leq C\left(\|\boldsymbol{f}\|_{\left[\mathbf{H}^{1}(\Omega)\right]^{\prime}}+\|g\|_{\mathrm{L}^{2}(\Omega)}+\left\|\boldsymbol{f}_{\boldsymbol{b}}\right\|_{\mathbf{H}^{-1 / 2}(\partial \Omega)}\right) .
$$

Moreover, for $k \geq 0$, if the open set $\Omega$ has a $C^{k+1,1}$ boundary and if

$$
\left(\boldsymbol{f}, g, \boldsymbol{f}_{\boldsymbol{b}}\right) \in \mathbf{H}^{k}(\Omega) \times \mathrm{H}^{k+1}(\Omega) \times \mathbf{H}^{k+\frac{1}{2}}(\partial \Omega),
$$

then the pair $(\boldsymbol{u}, p)$ belongs to $\mathbf{H}^{k+2}(\Omega) \times \mathrm{H}^{k+1}(\Omega)$, satisfies

$$
\left\{\begin{aligned}
-\nu \Delta \boldsymbol{u}+\nabla p & =\boldsymbol{f} \text { in } \Omega, \\
\operatorname{div} \boldsymbol{u} & =g \text { in } \Omega, \\
-\nu \partial_{\mathbf{n}} \boldsymbol{u}+p \mathbf{n} & =\boldsymbol{f}_{\boldsymbol{b}} \text { on } \partial \Omega,
\end{aligned}\right.
$$

and the following estimate holds:

$$
\|\boldsymbol{u}\|_{\mathbf{H}^{k+2}(\Omega)}+\|p\|_{\mathrm{H}^{k+1}(\Omega)} \leq C\left(\|\boldsymbol{f}\|_{\mathbf{H}^{k}(\Omega)}+\|g\|_{\mathrm{H}^{k+1}(\Omega)}+\left\|\boldsymbol{f}_{\boldsymbol{b}}\right\|_{\mathbf{H}^{k+\frac{1}{2}}(\partial \Omega)}\right) .
$$

Proof. Existence and uniqueness. For the proof of the first part of Appendix A.1, let us begin by studying the case of null divergence. According to Lax-Milgram's Theorem, there exists a unique $\boldsymbol{u} \in \boldsymbol{V}^{1}(\Omega) \cap \mathbf{L}_{0}^{2}(\Omega)$ such that

$$
\int_{\Omega} \nu \nabla \boldsymbol{u}: \nabla \boldsymbol{v}=\langle\boldsymbol{f}, \boldsymbol{v}\rangle_{\Omega}-\left\langle\boldsymbol{f}_{\boldsymbol{b}}, \boldsymbol{v}\right\rangle_{\partial \Omega} \quad \forall \boldsymbol{v} \in \boldsymbol{V}^{1}(\Omega) \cap \mathbf{L}_{0}^{2}(\Omega),
$$

and we have

$$
\|\boldsymbol{u}\|_{\mathbf{H}^{1}(\Omega)} \leq C\left(\|\boldsymbol{f}\|_{\left[\mathbf{H}^{1}(\Omega)\right]^{\prime}}+\left\|\boldsymbol{f}_{\boldsymbol{b}}\right\|_{\mathbf{H}^{-1 / 2}(\partial \Omega)}\right) .
$$

For $\boldsymbol{v} \in \boldsymbol{V}^{1}(\Omega)$, we define

$$
\boldsymbol{c}(\boldsymbol{v}):=\frac{1}{|\Omega|} \int_{\Omega} \boldsymbol{v}
$$

and we use $\boldsymbol{v}-\boldsymbol{c}(\boldsymbol{v}) \in \boldsymbol{V}^{1}(\Omega) \cap \mathbf{L}_{0}^{2}(\Omega)$ as a test function in (A.2). According to the compatibility condition, we check that (A.2) is also true for all $\boldsymbol{v} \in \boldsymbol{V}^{1}(\Omega)$ and in particular for all $\boldsymbol{v} \in \boldsymbol{V}^{1}(\Omega) \cap \mathbf{H}_{0}^{1}(\Omega)$. Then, using De Rham's Theorem (see for example Lemma 2.7 in Ref. 7 ), there exists $p \in \mathrm{L}^{2}(\Omega)$, up to an additive constant, such that

$$
\int_{\Omega} \nu \nabla \boldsymbol{u}: \nabla \boldsymbol{v}-\int_{\Omega} p \operatorname{div} \boldsymbol{v}=\left\langle\boldsymbol{f}_{\mid \mathbf{H}_{0}^{1}(\Omega)}, \boldsymbol{v}\right\rangle_{\mathbf{H}^{-1}(\Omega), \mathbf{H}_{0}^{1}(\Omega)} \forall \boldsymbol{v} \in \mathbf{H}_{0}^{1}(\Omega) .
$$


We define $\varphi_{N}(\boldsymbol{x}):={ }^{t}\left(x_{1}, \ldots, x_{N}\right) / N$, where $N$ is the dimension and $\left(x_{1}, \ldots, x_{N}\right)$ are the coordinates of $\boldsymbol{x}: \varphi_{N}$ is such that $\operatorname{div} \varphi_{N}=1$. Let $\boldsymbol{v} \in \mathbf{H}^{1}(\Omega)$ and

$$
c_{b}(\boldsymbol{v}):=\frac{1}{|\Omega|} \int_{\partial \Omega} \boldsymbol{v} \cdot \mathbf{n} .
$$

Using Theorem 3.2 in Ref. 17 or Lemma 3.3 in Ref. 7, we define $\boldsymbol{v}_{\mathbf{2}} \in \boldsymbol{V}^{1}(\Omega)$ in such a way that $\boldsymbol{v}=\boldsymbol{v}_{\mathbf{1}}+\boldsymbol{v}_{\mathbf{2}}+c_{b}(\boldsymbol{v}) \boldsymbol{\varphi}_{\boldsymbol{N}}$, where $\boldsymbol{v}_{\mathbf{1}} \in \mathbf{H}_{0}^{1}(\Omega)$ satisfies

$$
\operatorname{div} \boldsymbol{v}_{\mathbf{1}}=\operatorname{div}\left(\boldsymbol{v}-c_{b}(\boldsymbol{v}) \boldsymbol{\varphi}_{\boldsymbol{N}}\right) .
$$

According to (A.2) (which remains valid for $\boldsymbol{v} \in \boldsymbol{V}^{1}(\Omega)$ ) and (A.4) and choosing the additive constant for $p$ such that $\int_{\Omega} p=-\left\langle\boldsymbol{f}, \varphi_{N}\right\rangle_{\Omega}+\left\langle f_{b}, \varphi_{N}\right\rangle_{\partial \Omega}$, we check that

$$
\int_{\Omega} \nu \nabla \boldsymbol{u}: \nabla \boldsymbol{v}-\int_{\Omega} p \operatorname{div} \boldsymbol{v}=\langle\boldsymbol{f}, \boldsymbol{v}\rangle_{\Omega}-\left\langle\boldsymbol{f}_{\boldsymbol{b}}, \boldsymbol{v}\right\rangle_{\partial \Omega} .
$$

Therefore, there is a unique pair $(\boldsymbol{u}, p) \in\left[\boldsymbol{V}^{1}(\Omega) \cap \mathbf{L}_{0}^{2}(\Omega)\right] \times \mathrm{L}^{2}(\Omega)$ such that

$$
\int_{\Omega} \nu \nabla \boldsymbol{u}: \nabla \boldsymbol{v}-\int_{\Omega} p \operatorname{div} \boldsymbol{v}=\langle\boldsymbol{f}, \boldsymbol{v}\rangle_{\Omega}-\left\langle\boldsymbol{f}_{\boldsymbol{b}}, \boldsymbol{v}\right\rangle_{\partial \Omega} \quad \forall \boldsymbol{v} \in \mathbf{H}^{1}(\Omega) .
$$

Let $\boldsymbol{v}:=\widetilde{\boldsymbol{v}}+c(p) \boldsymbol{\varphi}_{\boldsymbol{N}}$, where

$$
c(p):=\frac{1}{|\Omega|} \int_{\Omega} p
$$

and $\widetilde{\boldsymbol{v}} \in \mathbf{H}_{0}^{1}(\Omega)$ is such that $\operatorname{div} \widetilde{\boldsymbol{v}}=p-c(p)$ and $\|\widetilde{\boldsymbol{v}}\|_{\mathbf{H}_{0}^{1}(\Omega)} \leq C\|p\|_{\mathrm{L}^{2}(\Omega)}$ (see Theorem 3.2 in Ref. 17 or Lemma 3.3 in Ref. 7). Using $\boldsymbol{v}$ in (A.5), and according to (A.3), we obtain

$$
\|\boldsymbol{u}\|_{\mathbf{H}^{1}(\Omega)}+\|p\|_{\mathrm{L}^{2}(\Omega)} \leq C\left(\|\boldsymbol{f}\|_{\left[\mathbf{H}^{1}(\Omega)\right]^{\prime}}+\left\|\boldsymbol{f}_{\boldsymbol{b}}\right\|_{\mathbf{H}^{-1 / 2}(\partial \Omega)}\right) .
$$

The first part of the theorem is proved for $g=0$. The case $g \neq 0$ is obtained by a lifting argument. Let us define

$$
\boldsymbol{u}_{\boldsymbol{g}}:=\widetilde{\boldsymbol{u}_{\boldsymbol{g}}}+c(g) \boldsymbol{\varphi}_{\boldsymbol{N}}-\frac{1}{|\Omega|} \int_{\Omega}\left(\widetilde{\boldsymbol{u}_{\boldsymbol{g}}}+c(g) \boldsymbol{\varphi}_{\boldsymbol{N}}\right) \in \mathbf{H}^{1}(\Omega),
$$

where

$$
c(g):=\frac{1}{|\Omega|} \int_{\Omega} g
$$

and where, according to Theorem 3.2 in Ref. 17 or Lemma 3.3 in Ref. 7, $\widetilde{\boldsymbol{u}_{\boldsymbol{g}}} \in \mathbf{H}_{0}^{1}(\Omega)$ is such that $\operatorname{div} \widetilde{\boldsymbol{u}_{\boldsymbol{g}}}=g-c(g)$ and $\left\|\widetilde{\boldsymbol{u}_{\boldsymbol{g}}}\right\|_{\mathbf{H}_{0}^{1}(\Omega)} \leq C\|g\|_{\mathrm{L}^{2}(\Omega)}$. Thus $\boldsymbol{u}_{\boldsymbol{g}}$ is such that

$$
\int_{\Omega} \boldsymbol{u}_{\boldsymbol{g}}=\mathbf{0}, \quad \operatorname{div} \boldsymbol{u}_{\boldsymbol{g}}=g \text { in } \Omega \quad \text { and } \quad\left\|\boldsymbol{u}_{\boldsymbol{g}}\right\|_{\mathbf{H}^{1}(\Omega)} \leq C\|g\|_{\mathrm{L}^{2}(\Omega)} .
$$

Thus, defining $\boldsymbol{u}_{0}:=\boldsymbol{u}-\boldsymbol{u}_{\boldsymbol{g}}$ and

$$
\widetilde{\boldsymbol{f}}: \boldsymbol{v} \in \mathbf{H}^{1}(\Omega) \mapsto\langle\boldsymbol{f}, \boldsymbol{v}\rangle_{\Omega}-\int_{\Omega} \nu \nabla \boldsymbol{u}_{\boldsymbol{g}}: \nabla \boldsymbol{v},
$$


the problem (A.1) is equivalent to

$$
\left\{\begin{array}{c}
\text { Find }\left(\boldsymbol{u}_{\mathbf{0}}, p\right) \in\left[\boldsymbol{V}^{1}(\Omega) \cap \mathbf{L}_{0}^{2}(\Omega)\right] \times \mathrm{L}^{2}(\Omega) \text { such that } \\
\int_{\Omega} \nu \nabla \boldsymbol{u}_{\mathbf{0}}: \nabla \boldsymbol{v}-\int_{\Omega} p \operatorname{div} \boldsymbol{v}=\langle\tilde{\boldsymbol{f}}, \boldsymbol{v}\rangle_{\Omega}-\left\langle\boldsymbol{f}_{\boldsymbol{b}}, \boldsymbol{v}\right\rangle_{\partial \Omega} \quad \forall \boldsymbol{v} \in \mathbf{H}^{1}(\Omega) .
\end{array}\right.
$$

Regularity of the solution. For the second part of Appendix A.1 concerning the regularity of $(\boldsymbol{u}, p)$, we refer to Section 5.3 in Ref. 9 and Ref. 18 for the homogeneous case $g=0$ and $\boldsymbol{f}_{\boldsymbol{b}}=\mathbf{0}$. To obtain the result for $g \neq 0$ and $\boldsymbol{f}_{\boldsymbol{b}} \neq \mathbf{0}$ let us define

$$
\boldsymbol{\varphi}_{g}:=\frac{1}{|\Omega|}\left(\int_{\Omega} g\right) \boldsymbol{\varphi}_{\boldsymbol{N}} \quad \text { and } \quad \Psi(g):=2 \nu \mathrm{H} \boldsymbol{\varphi}_{g} \cdot \mathbf{n}-\nu \operatorname{div}_{\Gamma}\left(\boldsymbol{\varphi}_{g}\right)_{\tau}
$$

where $\left(\boldsymbol{\varphi}_{g}\right)_{\tau}$ is the tangential component of $\boldsymbol{\varphi}_{g}$ and $\mathrm{H}$ is the mean curvature of $\partial \Omega$. According to Theorem A in Ref. 6, there exists $\widetilde{\boldsymbol{u}} \in \mathbf{H}^{k+2}(\Omega)$ such that

$$
\operatorname{div} \widetilde{\boldsymbol{u}}=g \text { in } \Omega, \quad \widetilde{\boldsymbol{u}}=\boldsymbol{\varphi}_{g} \quad \text { and } \quad \nu \partial_{\mathbf{n}} \widetilde{\boldsymbol{u}}=-\left(\boldsymbol{f}_{\boldsymbol{b}}\right)_{\tau}+\Psi(g) \mathbf{n}+\nu g \mathbf{n} \text { on } \partial \Omega,
$$

and which satisfies the following estimate:

$$
\|\widetilde{\boldsymbol{u}}\|_{\mathbf{H}^{k+2}(\Omega)} \leq C\left(\|g\|_{\mathrm{H}^{k+1}(\Omega)}+\left\|\boldsymbol{f}_{\boldsymbol{b}}\right\|_{\mathbf{H}^{k+1 / 2}(\partial \Omega)}\right) .
$$

Moreover, with a continuous right inverse of the trace operator, we define a pressure function $\widetilde{p} \in \mathrm{H}^{k+1}(\Omega)$ such that $\widetilde{p}=\boldsymbol{f}_{\boldsymbol{b}} \cdot \mathbf{n}+\Psi(g)+g$ on $\partial \Omega$ and which depends continuously on $\left(g, \boldsymbol{f}_{\boldsymbol{b}}\right)$ in $\mathrm{H}^{k+1}(\Omega) \times \mathbf{H}^{k+1 / 2}(\Omega)$. Then we have constructed a pair $(\widetilde{\boldsymbol{u}}, \widetilde{p})$ such that

$$
-\nu \partial_{\mathbf{n}} \widetilde{\boldsymbol{u}}+\widetilde{p} \mathbf{n}=\boldsymbol{f}_{\boldsymbol{b}} \text { on } \partial \Omega
$$

and which obeys the following estimate:

$$
\|\widetilde{\boldsymbol{u}}\|_{\mathbf{H}^{k+2}(\Omega)}+\|\widetilde{p}\|_{\mathrm{H}^{k+1}(\Omega)} \leq C\left(\|g\|_{\mathrm{H}^{k+1}(\Omega)}+\left\|\boldsymbol{f}_{\boldsymbol{b}}\right\|_{\mathbf{H}^{k+1 / 2}(\partial \Omega)}\right) .
$$

Finally, we check that the vector field $\boldsymbol{F}:=\boldsymbol{f}+\nu \Delta \widetilde{\boldsymbol{u}}-\nabla \widetilde{p} \in \mathbf{H}^{k}(\Omega)$ satisfies

$$
\|\boldsymbol{F}\|_{\mathbf{H}^{k}(\Omega)} \leq C\left(\|\boldsymbol{f}\|_{\mathbf{H}^{k}(\Omega)}+\|g\|_{\mathrm{H}^{k+1}(\Omega)}+\left\|\boldsymbol{f}_{\boldsymbol{b}}\right\|_{\mathbf{H}^{k+1 / 2}(\partial \Omega)}\right),
$$

and, since regularity results in the homogeneous case (see Ref. 9 and Ref. 18) ensures that the solution to:

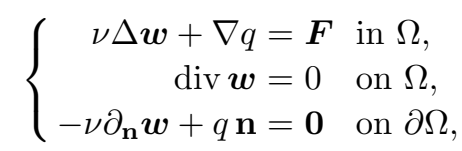

belongs to $\mathbf{H}^{k+2}(\Omega) \times \mathrm{H}^{k+1}(\Omega)$ and satisfies:

$$
\|\boldsymbol{w}\|_{\mathbf{H}^{k+2}(\Omega)}+\|q\|_{\mathrm{H}^{k+1}(\Omega)} \leq C\|\boldsymbol{F}\|_{\mathbf{H}^{k}(\Omega)},
$$

the conclusion follows by remarking that $(\boldsymbol{u}, p)=(\boldsymbol{w}+\widetilde{\boldsymbol{u}}, q+\widetilde{p})$ and by combining (A.6) with (A.7). 
Remark Appendix A.1. As in the proof of Appendix A.1, we can obtain the following result from Theorem A in Ref. 6: if $\Omega$ is a bounded connected open set of $\mathbb{R}^{N}$ with a $C^{k+1}$ boundary and if

$$
\left(g, \boldsymbol{b}_{\mathbf{0}}, \boldsymbol{b}_{\mathbf{1}}\right) \in \mathrm{H}^{k+1}(\Omega) \times \mathbf{H}^{k+3 / 2}(\partial \Omega) \times \mathbf{H}^{k+1 / 2}(\partial \Omega)
$$

satisfies $\int_{\partial \Omega} \boldsymbol{b}_{\mathbf{0}} \cdot \mathbf{n}=\int_{\Omega} g$, then there exists a pair $(\boldsymbol{v}, \pi) \in \mathbf{H}^{k+2}(\Omega) \times \mathrm{H}^{k+1}(\Omega)$ such that

$$
\operatorname{div} \boldsymbol{v}=g \text { in } \Omega, \quad \boldsymbol{v}=\boldsymbol{b}_{\mathbf{0}} \quad \text { and } \quad-\nu \partial_{\mathbf{n}} \boldsymbol{v}+\pi \mathbf{n}=\boldsymbol{b}_{\mathbf{1}} \text { on } \partial \Omega
$$

and

$$
\|\boldsymbol{v}\|_{\mathbf{H}^{k+2}(\Omega)}+\|\pi\|_{\mathrm{H}^{k+1}(\Omega)} \leq C\left(\|g\|_{\mathrm{H}^{k+1}(\Omega)}+\left\|\boldsymbol{b}_{\mathbf{0}}\right\|_{\mathbf{H}^{k+3 / 2}(\partial \Omega)}+\left\|\boldsymbol{b}_{\mathbf{1}}\right\|_{\mathbf{H}^{k+1 / 2}(\partial \Omega)}\right) .
$$

To enunciate the following result concerning the local regularity of the solution of the Stokes problem, we recall some notations introduced in section 3: for $k, m \in \mathbb{N}, k<m$ and for two open sets $\Omega_{1}$ and $\Omega_{2}$ such that $\Omega_{2} \subset \Omega_{1}$, we note $\mathrm{X}^{k, m}\left(\Omega_{1}, \Omega_{2}\right)$ the space of functions in $\mathrm{H}^{k}\left(\Omega_{1}\right)$ such that their restriction to $\Omega_{2}$ belongs to $\mathrm{H}^{m}\left(\Omega_{2}\right)$. Similarly, $\mathrm{X}^{*, m}\left(\Omega_{1}, \Omega_{2}\right)$ is the space of functions in $\left[\mathrm{H}^{1}\left(\Omega_{1}\right)\right]^{\prime}$ such that their restriction to $\Omega_{2}$ belongs to $\mathrm{H}^{m}\left(\Omega_{2}\right)$.

Theorem Appendix A.2 (Local regularity). Let $k \in \mathbb{N}, \nu>0, \Omega$ a bounded connected Lipschitz open set of $\mathbb{R}^{N}\left(N \in \mathbb{N}^{*}\right)$ and $\omega$ an open set with a $C^{k+1,1}$ boundary strictly included in $\Omega$ (that is to say there exists $\delta>0$ such that $\omega$ is at least at distance $\delta$ of the boundary $\partial \Omega$ ) and such that $\Omega \backslash \bar{\omega}$ is connected. Let $\mathcal{C}$ and $\mathcal{C}^{\prime}$ two smooth open subsets of $\Omega \backslash \bar{\omega}$ such that $\partial \omega \subset \partial \mathcal{C}, \partial \omega \subset \partial \mathcal{C}^{\prime}, \overline{\mathcal{C}} \subset \mathcal{C}^{\prime}$ and $\overline{\mathcal{C}^{\prime}} \subset \Omega$. Let

$$
\left(\boldsymbol{f}, g, \boldsymbol{h}_{\text {ext }}, \boldsymbol{h}_{\boldsymbol{i n t}}\right) \in \mathbf{X}^{*, k}\left(\Omega \backslash \bar{\omega}, \mathcal{C}^{\prime}\right) \times \mathrm{X}^{0, k+1}\left(\Omega \backslash \bar{\omega}, \mathcal{C}^{\prime}\right) \times \mathbf{H}^{-1 / 2}(\partial \Omega) \times \mathbf{H}^{k+\frac{1}{2}}(\partial \omega),
$$

such that $\int_{\Omega \backslash \bar{\omega}} f^{i}=\left\langle h_{\text {ext }}^{i}, 1\right\rangle_{\partial \Omega}+\int_{\partial \omega} h_{\text {int }}^{i}$ for all $i=1, \ldots, N$, where $f^{i}, h_{\text {ext }}^{i}$ and $h_{\text {int }}^{i}$ are the respective components of $\boldsymbol{f}, \boldsymbol{h}_{\boldsymbol{e x t}}$ and $\boldsymbol{h}_{\boldsymbol{i n t}}$. We consider $(\boldsymbol{u}, p)$ the solution in $\mathbf{H}^{1}(\Omega \backslash \bar{\omega}) \times \mathrm{L}^{2}(\Omega \backslash \bar{\omega})$ of the following Stokes problem with Neumann boundary conditions

$$
\left\{\begin{aligned}
-\nu \Delta \boldsymbol{u}+\nabla p & =\boldsymbol{f} & & \text { in } \Omega \backslash \bar{\omega} \\
\operatorname{div} \boldsymbol{u} & =g & & \text { in } \Omega \backslash \bar{\omega} \\
-\nu \partial_{\mathbf{n}} \boldsymbol{u}+p \mathbf{n} & =\boldsymbol{h}_{\text {ext }} & & \text { on } \partial \Omega \\
-\nu \partial_{\mathbf{n}} \boldsymbol{u}+p \mathbf{n} & =\boldsymbol{h}_{\boldsymbol{i n t} \boldsymbol{t}} & & \text { on } \partial \omega .
\end{aligned}\right.
$$

Then $(\boldsymbol{u}, p)$ belongs to $\mathbf{H}^{k+2}(\mathcal{C}) \times \mathrm{H}^{k+1}(\mathcal{C})$ and the following estimate holds:

$$
\begin{aligned}
& \|\boldsymbol{u}\|_{\mathbf{H}^{k+2}(\mathcal{C})}+\|p\|_{\mathrm{H}^{k+1}(\mathcal{C})} \\
\leq & C\left(\|\boldsymbol{f}\|_{\mathbf{X}^{*, k}\left(\Omega \backslash \bar{\omega}, \mathcal{C}^{\prime}\right)}+\|g\|_{\mathbf{X}^{0, k+1}\left(\Omega \backslash \bar{\omega}, \mathcal{C}^{\prime}\right)}+\left\|\boldsymbol{h}_{\boldsymbol{i n t} \boldsymbol{t}}\right\|_{\mathbf{H}^{k+\frac{1}{2}}(\partial \omega)}+\left\|\boldsymbol{h}_{\boldsymbol{e x t} \boldsymbol{t}}\right\|_{\mathbf{H}^{-1 / 2}(\partial \Omega)}\right) .
\end{aligned}
$$


Proof. First, let us consider the case $k=0$. We define $\mathcal{V}:=\mathcal{C} \cup \bar{\omega}, \mathcal{V}^{\prime}:=\mathcal{C}^{\prime} \cup \bar{\omega}$ and $\varphi \in C_{c}^{\infty}(\Omega)$ such that $0 \leq \varphi \leq 1, \varphi \equiv 1$ in $\mathcal{V}$ and $\varphi \equiv 0$ in $\Omega \backslash \mathcal{V}^{\prime}$. Let

$$
(\boldsymbol{u}, p) \in\left[\mathbf{H}^{1}(\Omega \backslash \bar{\omega}) \cap \mathbf{L}_{0}^{2}(\Omega \backslash \bar{\omega})\right] \times \mathrm{L}^{2}(\Omega \backslash \bar{\omega})
$$

the solution of problem (A.8) given by Appendix A.1. Using (A.8), we check that

$$
\left\{\begin{aligned}
-\nu \Delta(\varphi \boldsymbol{u})+\nabla(\varphi p) & =\widetilde{\boldsymbol{f}} & & \text { in } \mathcal{C}^{\prime} \\
\operatorname{div}(\varphi \boldsymbol{u}) & =\widetilde{g} & & \text { in } \mathcal{C}^{\prime}, \\
-\nu \partial_{\mathbf{n}}(\varphi \boldsymbol{u})+(\varphi p) \mathbf{n} & =\mathbf{0} & & \text { on } \partial \mathcal{V}^{\prime} \\
-\nu \partial_{\mathbf{n}}(\varphi \boldsymbol{u})+(\varphi p) \mathbf{n} & =\boldsymbol{h}_{\text {int }} & & \text { on } \partial \omega
\end{aligned}\right.
$$

where $\tilde{\boldsymbol{f}}:=\varphi \boldsymbol{f}-\nu \boldsymbol{u} \Delta \varphi-2 \nu \nabla \boldsymbol{u} \nabla \varphi+p \nabla \varphi \in \mathbf{L}^{2}\left(\mathcal{C}^{\prime}\right)$ and $\widetilde{g}:=\varphi g+\boldsymbol{u} \cdot \nabla \varphi \in \mathrm{H}^{1}\left(\mathcal{C}^{\prime}\right)$. From Appendix A.1, $\varphi \boldsymbol{u} \in \mathbf{H}^{2}\left(\mathcal{C}^{\prime}\right), \varphi p \in \mathrm{H}^{1}\left(\mathcal{C}^{\prime}\right)$ and, taking into account of the expression of $\widetilde{\boldsymbol{f}}$ and $\widetilde{g}$, we also have

$$
\begin{aligned}
& \|\varphi \boldsymbol{u}\|_{\mathbf{H}^{2}\left(\mathcal{C}^{\prime}\right)}+\|\varphi p\|_{\mathrm{H}^{1}\left(\mathcal{C}^{\prime}\right)} \\
& \leq C\left(\|\varphi \boldsymbol{f}\|_{\mathbf{L}^{2}\left(\mathcal{C}^{\prime}\right)}+\|\varphi g\|_{\mathrm{H}^{1}\left(\mathcal{C}^{\prime}\right)}+\|\boldsymbol{u}\|_{\mathbf{H}^{1}(\Omega \backslash \bar{\omega})}+\|p\|_{\mathrm{L}^{2}(\Omega \backslash \bar{\omega})}+\left\|\boldsymbol{h}_{\boldsymbol{i n t}}\right\|_{\mathbf{H}^{1 / 2}(\partial \omega)}\right) .
\end{aligned}
$$

Using this inequality and the estimate on $\|\boldsymbol{u}\|_{\mathbf{H}^{1}(\Omega \backslash \bar{\omega})}$ and $\|p\|_{\mathrm{L}^{2}(\Omega \backslash \bar{\omega})}$ given by Appendix A.1, we obtain the announced estimate for $k=0$. Proceeding as above, we conclude by induction.

\section{References}

1. R. A. Adams and J. J. F. Fournier. Sobolev spaces, volume 140 of Pure and Applied Mathematics (Amsterdam). Elsevier/Academic Press, Amsterdam, second edition, 2003.

2. L. Afraites, M. Dambrine, K. Eppler, and D. Kateb. Detecting perfectly insulated obstacles by shape optimization techniques of order two. Discrete Contin. Dyn. Syst. Ser. B, 8(2):389-416 (electronic), 2007.

3. L. Afraites, M. Dambrine, and D. Kateb. On second order shape optimization methods for electrical impedance tomography. SIAM J. Control Optim., 47(3):1556-1590, 2008.

4. C. Alvarez, C. Conca, L. Friz, O. Kavian, and J. H. Ortega. Identification of immersed obstacles via boundary measurements. Inverse Problems, 21(5):1531-1552, 2005.

5. C. J. S. Alves, R. Kress, and A. L. Silvestre. Integral equations for an inverse boundary value problem for the two-dimensional Stokes equations. J. Inverse Ill-Posed Probl., 15(5):461-481, 2007.

6. C. Amrouche and V. Girault. Problèmes généralisés de Stokes. Portugal. Math., 49(4):463-503, 1992.

7. C. Amrouche and V. Girault. Decomposition of vector spaces and application to the Stokes problem in arbitrary dimension. Czechoslovak Math. J., 44(119)(1):109-140, 1994.

8. A. Ballerini. Stable determination of an immersed body in a stationary stokes fluid. Inverse Problems, 26(12), 2010.

9. F. Boyer and P. Fabrie. Éléments d'analyse pour l'étude de quelques modèles d'écoulements de fluides visqueux incompressibles, volume 52 of Mathématiques $\mathcal{G}_{3}$ Applications (Berlin) [Mathematics 6 Applications]. Springer-Verlag, Berlin, 2006. 
10. D. Bucur and G. Buttazzo. Variational methods in shape optimization problems. Progress in Nonlinear Differential Equations and their Applications, 65. Birkhäuser Boston Inc., Boston, MA, 2005.

11. C. Conca, P. Cumsille, J. Ortega, and L. Rosier. Detecting a moving obstacle in an ideal fluid by a boundary measurement. C. R. Math. Acad. Sci. Paris, 346(15-16):839844, 2008.

12. C. Conca, M. Malik, and M. Alexandre. On the detection of a moving rigid solid in a perfect fluid. Inverse Problems, 26(9), 2010, DOI=10.1088/0266-5611/26/9/095010.

13. M. Dambrine. On variations of the shape Hessian and sufficient conditions for the stability of critical shapes. RACSAM Rev. R. Acad. Cienc. Exactas Fis. Nat. Ser. A Mat., 96(1):95-121, 2002.

14. K. Eppler and H. Harbrecht. A regularized Newton method in electrical impedance tomography using shape Hessian information. Control Cybernet., 34(1):203-225, 2005.

15. L. C. Evans. Partial differential equations, volume 19 of Graduate Studies in Mathematics. American Mathematical Society, Providence, RI, 1998.

16. C. Fabre and G. Lebeau. Prolongement unique des solutions de l'equation de Stokes. Comm. Partial Differential Equations, 21(3-4):573-596, 1996.

17. G. P. Galdi. An introduction to the mathematical theory of the Navier-Stokes equations. Vol. I, volume 38 of Springer Tracts in Natural Philosophy. Springer-Verlag, New York, 1994. Linearized steady problems.

18. G. Grubb and V. A. Solonnikov. Boundary value problems for the nonstationary Navier-Stokes equations treated by pseudo-differential methods. Math. Scand., 69(2):217-290 (1992), 1991.

19. H. Heck, G. Uhlmann, and J.-N. Wang. Reconstruction of obstacles immersed in an incompressible fluid. Inverse Probl. Imaging, 1(1):63-76, 2007.

20. A. Henrot and M. Pierre. Variation et optimisation de formes, volume 48 of Mathématiques \& Applications (Berlin) [Mathematics \& A Applications]. Springer, Berlin, 2005. Une analyse géométrique. [A geometric analysis].

21. V. Maz'ya and T. Shaposhnikova. Theory of multipliers in spaces of differentiable functions, volume 23 of Monographs and Studies in Mathematics. Pitman (Advanced Publishing Program), Boston, MA, 1985.

22. F. Murat and J. Simon. Sur le contrôle par un domaine géométrique. Rapport du L.A. 189, 1976. Université de Paris VI, France.

23. J. Simon. Domain variation for drag in Stokes flow. In Control theory of distributed parameter systems and applications (Shanghai, 1990), volume 159 of Lecture Notes in Control and Inform. Sci., pages 28-42. Springer, Berlin, 1991.

24. R. Temam Navier-Stokes equations, Theory and numerical analysis, Reprint of the 1984 edition. AMS Chelsea Publishing, Providence, RI, 2001 